\title{
How Well Do Markov Switching Models Describe Actual Business Cycles? The Case of Synchronization*
}

\author{
Penelope A. Smith and Peter M. Summers \\ Melbourne Institute of Applied Economic and Social Research \\ The University of Melbourne
}

Melbourne Institute Working Paper No. 9/04

ISSN 1328-4991 (Print)

ISSN 1447-5863 (Online)

ISBN 0734031513

May 2004

\begin{abstract}
*We are grateful to Dick van Dijk, Don Harding, Adrian Pagan, Christopher Sims, and two referees for many helpful comments, but retain all responsibility for any remaining errors. This work was sponsored in part by Australian Research Council grant C79930704.
\end{abstract}

Melbourne Institute of Applied Economic and Social Research

The University of Melbourne

Victoria 3010 Australia

Telephone (03) 83442100

Fax (03) 83442111

Email melb-inst@unimelb.edu.au

WWW Address http://www.melbourneinstitute.com 


\begin{abstract}
The objective of this paper is to evaluate the effectiveness of using a Markov switching model to measure the synchronization of business cycles. We use a Bayesian, Gibbs sampling approach to estimate a multivariate Markov switching model of GDP growth for several countries. We look for evidence of synchronization across countries in the sense of common Markov states, covariance of impulses and a long-run co-integrating relationship. We then use the fitted data implied by the posterior distribution of the Markov switching VAR, in conjunction with a dating rule, to obtain the posterior distribution of binary business cycle states. We use these to investigate the posterior distributions of non-parametric measures of synchronization described by Harding and Pagan (2003) and compare them with similar measures obtained from standard reference chronologies. As a point of reference, we repeat this exercise using simulated data from a linear VAR.

We find no evidence of a common Markov state, but some evidence of the propagation of country-specific disturbances across countries and of a co-integrating relationship between the United States and Canada. Posterior odds ratios overwhelmingly favor the Markov switching model over the linear VAR and we find that the posterior distributions of the non-parametric measures of synchronisation produced by the Markov switching VAR match the data more closely than those produced by the linear VAR.
\end{abstract}




\section{Introduction and motivation}

Since James Hamilton's seminal 1989 Econometrica paper, numerous studies have applied Markov switching models to the study of business cycles. Among many others, see McCulloch and Tsay (1994), Albert and Chib (1993), Durland and McCurdy (1994), Filardo (1994), Goodwin (1993), Kim and Nelson (1999a, b), and McConnell and Perez-Quiros (2000). Within this framework output growth is modelled as an autoregressive process subject to parameter shifts, usually in the conditional mean, as determined by a latent Markov process. Markov switching models are able to capture asymmetries in the depth and duration of output growth phases and, in general, are able to fit the data better than linear models of output growth.

As Markov switching models provide information about the timing of regime changes, they are often used to create business cycle chronologies, and these can be used to measure synchronization (see, for example Bodman and Crosby (2000), Guha and Banerji (1998)). However, a univariate regime switching framework seems an inappropriate tool for drawing inferences about the synchronization of business cycles, as estimating individual Markov switching models for each country is equivalent to imposing the restriction that the data are drawn from a mixture of normal distributions with a diagonal covariance matrix.

Literature on the nature of synchronization between international business cycles using a multivariate regime switching framework has been quite limited. Phillips (1991) estimates a bivariate version of Hamilton's (1989) regime switching model in which the unconditional means of real GDP growth for a pair of economies are driven by an unobserved four state Markov process: a high growth and low growth state for each country. Evidence of synchronization is assessed by testing restrictions on the transition matrix which would imply a common two state Markov process affecting 
output growth in each country.

Krolzig (1997) extends Phillips' (1991) analysis to the multivariate case, assuming perfect synchronization of Markov states across countries. This assumption is made in order to make the analysis tractable as an unrestricted version of an $n$-country model with $2^{n}$ possible regimes would require the estimation of $n^{2}$ transition probabilities. Any synchronization of business cycles in this approach arises through common shocks as measured by the off-diagonal elements of the covariance matrix and through the propagation of cross country and country-specific shocks through the vector autoregressive terms. Artis et al. (2004), examining the European business cycle, extend Krolzig's (1997) analysis to allow for the possibility of a long run cointegrating relationship amongst the series.

An alternative framework which avoids the restrictive assumption of a common Markov state is developed by Paap and Dijk (2003), who modelled the relationship between consumption and income. This approach allows the growth rate in each country to depend on both its own Markov state and the Markov states of other countries. In this approach only $2 n$ transition probabilities need be estimated.

In the first part of our paper we adapt the Bayesian methods of Paap and van Dijk to investigate the posterior distributions of the parameters of a six country Markov switching vector autoregression (MS-VAR), in order to examine any synchronization that occurs in international business cycles. We look for evidence of a common Markov state and for evidence of a long run cointegrating relationship in the countries' GDP data. In addition we examine the extent to which countries are affected by common impulses and the nature of the propagation of these impulses between countries. For comparison, we also obtain the posterior distributions of a linear VAR and use posterior odds ratios to test the hypothesis of linearity. 
Harding and Pagan (2002) and Hess and Iwata (1997) have recently questioned the appropriateness of using Markov switching models to capture the features of the business cycle. In order to evaluate several popular models of real GDP growth, including Markov switching models, they simulate data from the estimated parameters of the models and compare the ability of the simulated data to match the cyclical characteristics of the observed data, such as the duration and amplitude of phases of the classical business cycle. Amongst their findings is that find that Markov switching models perform quite poorly in this regard relative to a simple AR(1) model.

In the second part of our paper, we seek to examine whether or not allowing for Markov switches in each country's conditional mean growth rate provides any information about the synchronization of business cycles over and above the linear VAR. To do so, we make use of the measures of synchronization proposed by Harding and Pagan (2003) who investigate the nature of what "synchronization" means in the context of binary time series, drawing a distinction between concordance (the fraction of time that two series are in the same state) and correlation (the extent to which turning points in the two series occur near each other). By using the fitted data implied by the posterior distributions of the parameters of the VAR and the MS-VAR along with random innovations, we construct the posterior distributions of concordance and correlation statistics and compare them with the concordance and correlation of reference chronologies for classical cycles produced by the National Bureau of Economic Research (NBER) and the Economic Cycle Research Institute (ECRI) .

When assessing the fit of the two models to the data, we demonstrate that it is important to distinguish measures of fit for which the characteristics of turning points implied by a model (such as duration or amplitude) are key, and measures for which it is the location of turning points that matters. The main distinction between our Markov 
switching and linear VAR models is along this dimension. Although the linear VAR produces turning points that agree fairly well with the data, we demonstrate that the MS-VAR model contains vital information about the likely location of these turning points. The posterior distribution of turning points generated by the linear VAR is essentially flat across the sample period; as a consequence, the modal value of the correlation of business cycles is zero for all country pairs.

\section{Dating cycles}

We base our model on Paap and Dijk (2003), decomposing the vector of real GDP

levels $Y_{t}=\log \left(y_{t}^{1}, \ldots, y_{t}^{n}\right)^{\prime}$ into a trend component $N_{t}$ and a $\operatorname{VAR}(k)$ process, $Z_{t}$

$$
Y_{t}=N_{t}+Z_{t}
$$

where

$$
Z_{t}=\sum_{i=1}^{k} \Phi_{i} Z_{t-i}+\varepsilon_{t}
$$

with $\varepsilon_{t} \sim N(0, \Sigma)$.

The trend component is assumed to be influenced by the unobserved state of each economy, $S_{t}^{i}$, modeled as an independent, first order, Markov process, with $S_{t}^{i}=0$ indicating a period of high trend growth and $S_{t}^{i}=1$ indicating a period of low trend growth for country $i$. Each Markov process is assumed to be stationary and ergodic, switching according to the transition probabilities

$$
\begin{aligned}
& \operatorname{Pr}\left(S_{t}^{i}=0 \mid S_{t-1}^{i}=0\right)=p_{i} \\
& \operatorname{Pr}\left(S_{t}^{i}=1 \mid S_{t-1}^{i}=1\right)=q_{i} .
\end{aligned}
$$

The trend is modelled as

$$
N_{t}=N_{t-1}+\Gamma_{0}+\Gamma_{1} S_{t}
$$


where $\Gamma_{0}=\left(\Gamma_{0}^{1}, \ldots, \Gamma_{0}^{n}\right)^{\prime}$ and $S_{t}=\left(S_{t}^{1}, \ldots, S_{t}^{n}\right)^{\prime}$ are $n \times 1$ vectors and $\Gamma_{1}$ is an $n \times n$ matrix so that an individual country's trend component may be influenced by own its own Markov state $S_{t}^{i}$ as well as the Markov states of other countries, $S_{t}^{j} i \neq j$. As described by Paap and Dijk (2003), the backward solution of (2) is

$$
N_{t}=N_{1}+N_{t-1}+\Gamma_{0}(t-1)+\Gamma_{1} \sum_{i=2}^{t} S_{i}
$$

where $N_{1}$ is the initial value of the Markov trend.

It is by now well known that any $V A R(k)$ process of form (1) may be expressed equivalently as

$$
\Delta Z_{t}=\Pi Z_{t-1}+\sum_{i=1}^{k-1} \bar{\Phi}_{i} \Delta Z_{t-i}+\varepsilon_{t}
$$

where $\Pi=\sum_{j=1}^{k} \Phi_{j}-I_{n}$ and $\bar{\Phi}_{i}=\sum_{j=i+1}^{k-1} \Phi_{j}, i=1, \ldots, k-1$. The model is stationary if all the solutions to

$$
\left|I_{n}-\Phi_{1} z-\ldots-\Phi_{k} z^{k}\right|=0
$$

lie outside the unit circle. In this case $Z_{t}$ and therefore $Y_{t}$ will be stationary around a multivariate Markov trend and the long run impact matrix $\Pi$ will be of full rank; $\operatorname{rank}(\Pi)=n$. As discussed by Paap and Dijk (2003), this will be a multivariate extension of the univariate Markov switching model of Lam (1990) and may be expressed in full as

$$
\begin{aligned}
\left(\Delta Y_{t}-\Gamma_{0}-\Gamma_{1} S_{t}\right)= & \Pi\left(Y_{t-1}-N_{1}-\Gamma_{0}(t-2)-\Gamma_{1} \sum_{i=2}^{t-1} S_{i}\right) \\
& +\sum_{i=1}^{k-1} \bar{\Phi}_{i}\left(\Delta Y_{t-i}-\Gamma_{0}-\Gamma_{1} S_{t-i}\right)+\varepsilon_{t} .
\end{aligned}
$$

A second possibility is that $\Pi=0$, implying $n$ unit root solutions to (5) with remaining roots outside the unit circle. $Y_{t}$ is $I(1)$ and there is no long run relationship between the variables. In this case(4) may be expressed as

$$
\left(\Delta Y_{t}-\Gamma_{0}-\Gamma_{1} S_{t}\right)=\sum_{i=1}^{k-1} \bar{\Phi}_{i}\left(\Delta Y_{t-i}-\Gamma_{0}-\Gamma_{1} S_{t-i}\right)+\varepsilon_{t},
$$


which is a multivariate extension of the model of Hamilton (1989).

A third case of interest occurs when $0<\operatorname{rank}(\Pi)=r<n$ implying that $n-r$ roots lie on the unit circle, while other roots lie outside the unit circle. There will then be $n-r$ common stochastic trends. In this case $\Pi$ can be expressed as the product $\alpha \beta^{\prime}$, where $\alpha$ and $\beta$ are $n \times r$ matrices of rank $r$ and $\beta^{\prime} Z_{t}$ is a stationary process. The matrix $\beta$ characterizes the $r$ cointegrating relationships between the elements of $Z_{t}$ and $\alpha$ describes the impact of of the cointegrating vectors on the evolution of $\Delta Z_{t}$. In this case (4) may be expressed as

$$
\begin{aligned}
\left(\Delta Y_{t}-\Gamma_{0}-\Gamma_{1} S_{t}\right)= & \alpha \beta^{\prime}\left(Y_{t-1}-N_{1}-\Gamma_{0}(t-2)-\Gamma_{1} \sum_{i=2}^{t-1} S_{i}\right) \\
& +\sum_{i=1}^{k-1} \bar{\Phi}_{i}\left(\Delta Y_{t-i}-\Gamma_{0}-\Gamma_{1} S_{t-i}\right)+\varepsilon_{t} .
\end{aligned}
$$

There are three sources of co-movement of real output series in the general model: Covariance of the VAR error terms, the propagation of impulses across countries via the $\Pi$ and $\bar{\Phi}$ terms, and through the common component of Markov level shifts in growth rates of real GDP as represented by $\Gamma_{1}$. Movements in output growth across countries will be independent if $\Pi, \bar{\Phi}, \Gamma_{1}$ and $\Sigma$ are diagonal.

\subsection{Estimation}

A drawback of estimating Markov switching models via maximum likelihood techniques is that they require a degree of approximation when making inferences about $S_{t}$. To see this note that as the state variables are unobserved, estimation of the model is a two stage process. In the first stage, the vector of unknown parameters $\theta=\left(p, q, \Phi(L), \Gamma_{0}, \Gamma_{1}, N_{0}, \Sigma\right)$ would be estimated so as to maximize the log of the unconditional density of $Y_{t}$. This is found to be the sum of the joint distributions across all possible states. Once estimates $\widehat{\theta}$ of $\theta$ have been obtained, inference about the probability of being in a particular state at a given point in time may be made by using the 
definition of conditional probability:

$$
\operatorname{Pr}\left(S_{t}=j \mid Y_{t} ; \widehat{\theta}\right)=p\left(Y_{t}, S_{t}=j ; \widehat{\theta}\right) f\left(Y_{t} ; \widehat{\theta}\right) .
$$

Therefore, estimates of the states do not reflect the uncertainty inherent in the estimates of $\widehat{\theta}$. Unlike the classical approach, Bayesian analysis treats both the parameters of the model and the unobserved states as random variables, with inference about $S_{t}$ drawn from their joint distribution conditional upon the data, $p\left(S_{t}, \theta \mid Y_{t}\right)$ rather than the conditional distribution, $\operatorname{Pr}\left(S_{t}=j \mid Y_{t} ; \widehat{\theta}\right)$. As noted by Albert and Chib (1993), this allows us to treat the unobserved states, $\left\{S_{t}\right\}_{t=1}^{T}$, as additional parameters to be estimated (through simulation), along with the unknown parameters, $\theta$.

We adopt the Bayesian approach for estimating the parameters of the model outlined in Paap and Dijk (2003). This procedure follows from recent work by Albert and Chib (1993), who demonstrated that Bayesian estimation of Markov switching models is relatively simple to implement using the Gibbs sampler.

We make only two departures from Paap and Dijk (2003). The first is related to how we identify the low growth states in the model. It is obvious that the likelihood associated with any Markov switching model is only identified up to a relabeling of the state-dependent parameters of the model. We associate the event $S_{t}=1$ with a period of recession by imposing the identifying restriction.

$$
\operatorname{diag}\left(\Gamma_{0}+\Gamma_{1}\right) \leq 0
$$

where $\operatorname{diag}(A)$ refers to the diagonal elements of the matrix $A$. This may appear to be an overly restrictive condition as it does not allow for the possibility of associating the event $S_{t}=1$ with growth recessions. ${ }^{1}$ In Smith and Summers (2003), following from recent work by Hamilton et al. (2003), we show that relying on an order restriction

\footnotetext{
${ }^{1}$ That is, periods where economic growth is below its trend rate, but still positive.
} 
for the means, in this case the weaker restriction $\operatorname{diag}\left(\Gamma_{1}\right) \leq 0$, can result in bimodal posterior distributions for key parameters in the model (such as the diagonal elements of $\Gamma_{0}$ and $\Gamma_{1}$ ). This bimodality can in turn distort the inference one draws about the fit of the model. We encountered similar problems when we experimented with allowing for growth cycles rather than classical business cycles in the multivariate framework presented here.

In order to sample $\Gamma_{1}$ so that $\operatorname{diag}\left(\Gamma_{1}\right) \leq-\operatorname{diag}\left(\Gamma_{0}\right)$ we adopt the approach of Geweke (1991), who suggested an efficient method for drawing samples from a truncated multivariate normal distribution subject to linear constraints. This method makes use of the property that the distribution of each element of $\Gamma_{1}$, conditional on each of the other elements of $\Gamma_{1}$, will also be truncated normal. As such, it is possible to sample recursively from univariate truncated normals subsequent to a Cholesky decomposition. This method was independently developed by Keane (1990), and Hajivassiliou and McFadden (1998). The resulting technique is known as the Gewke-HajivassiliouKeane (GHK) simulator. While several other methods for sampling from a truncated multivariate normal distribution exist, Hajivassiliou et al. (1996) make a comparison of several methods and conclude that the GHK simulator is the most reliable method. We adopt that approach here. ${ }^{2}$

The second difference between our approach and that of Paap and Dijk (2003) is that we specify independent beta priors for $p_{i}$ and $q_{i}$ rather than uniform priors. The transition probabilities in univariate Markov switching models contain important information about the expected duration of regimes. It can be easily shown that the expected duration of a high-growth regime for country $i$ is $\left(1-p_{i}\right)^{-1}$, while the expected duration of a low-growth regime is $\left(1-q_{i}\right)^{-1}$. Obviously, these parameters have a slightly

\footnotetext{
${ }^{2}$ Code for the simulator was obtained from the home page of V. Hajivassiliou http://econ.lse.ac.uk/ $/$ vassilis/
} 
different interpretation in the MS-VAR as the growth rate of country $i$ may also be affected (either positively or negatively) by low growth periods of the other countries. However, given that the prior means of the off-diagonal elements of $\Gamma_{1}, \Phi, \Pi$ and $\Sigma$ are set to zero, implying independence of business cycles, it seems reasonable to set the priors on $p_{i}$ and $q_{i}$ to be to consistent with business cycle frequencies. This is generally considered to be the case if transition probabilities lie in the interval 0.75-0.95 for quarterly data, implying business cycle durations in the range of 1-5 years. The prior distributions of $p_{11}$ and $p_{00}$ are accordingly set to have means of 0.8 and standard deviations of 0.16 for each country. These priors have been used often in Bayesian estimation of Markov switching models: see Kim and Nelson (1999b) or Albert and Chib (1993), for example.

\section{Posterior distributions}

Seasonally adjusted real GDP data for Australia, Canada, Japan, Germany the U.K. and the U.S. were taken from Datastream for the period 1961:I to 2001:IV. Thus $Y_{t}=\log \left(y_{t}^{A U S}, y_{t}^{U S A}, y_{t}^{U K}, y_{t}^{G E R}, y_{t}^{C A N}, y_{t}^{J P N}\right)^{\prime}$. To take account of the effect of German reunification we ran a de-meaning regression of German GDP growth on a dummy variable which took the value 1 in 1991:I, then adjusted the level series accordingly. Most studies show that $\mathrm{AR}(1)$ specification is sufficient to model the growth rate of real GDP so we set $k=2$. We generate 250,000 iterations of the Gibbs sampler and use the final 75,000 for inference. The point estimates of the posterior means of the parameters of equations (4), (3) and $\Sigma$ under the restriction $\Gamma_{1}=0$ and for the MS-VAR are presented in appendix 1. MS-VAR are presented in appendix 1. Parameters which are "significant" in the sense that their posterior mean is at least two posterior standard errors from zero 0 are printed in bold. ${ }^{3}$

\footnotetext{
${ }^{3}$ The "t-ratios" for residual variance estimates for Australia and Canada are 1.65 and 1.52, respectively
} 
Very few off-diagonal elements of the long-run impact matrix $\Pi$ are significant and the impact of a Markov trend has very little impact: the effect of the $Z_{t-1}^{U S}$ on $\Delta Z_{t}^{C A N}$ becomes significant, while the significance of the effect of $Z_{t-1}^{U S}$ on $\Delta Z_{t}^{A U S}$ falls away. The impact of the Markov trend on the short -run impact matrix $\bar{\Phi}$ is also quite limited, the only notable effect being that the significance of the impact of $\Delta Z_{t-1}^{U S}$ on $\Delta Z_{t}^{G E R}$ is removed, as is the impact of $\Delta Z_{t-1}^{G E R}$ on $\Delta Z_{t}^{J P N}$. The most striking effect of the inclusion of Markov trend on the estimated equation is the diagonalization of the covariance matrix $\Sigma$ and the reduction of the variance of country specific impulses for all countries except Australia.

The parameters of the $\Gamma_{1}$ matrix are best understood in conjunction with figure 1 below, which presents the posterior probabilities $P\left(S_{t}=1\right)$ for each country. Shaded areas represent classical recessions as dated by the NBER for the United States and ECRI for other countries. For each country except the United Kingdom and Australia, periods for which $P\left(S_{t}=1\right)>0.5$ seem to be associated with specific periods of recession.

\subsection{Posterior odds}

A by-product of our estimation procedure is an estimate of the posterior odds ratio in favor of the linear VAR relative to the MS-VAR. Newton and Raftery's (1994) method is used to estimate the marginal likelihoods of each model based upon the output of the posterior simulators. The log of the estimated marginal likelihood is 348.7053 for the MS-VAR model, while that of the linear VAR is 128.2073 . This results in a Bayes factor of 220.498 favouring MS-VAR, which, on the scale suggested by Kass and Raftery (1995), may be interpreted as decisive evidence against the linear VAR. 

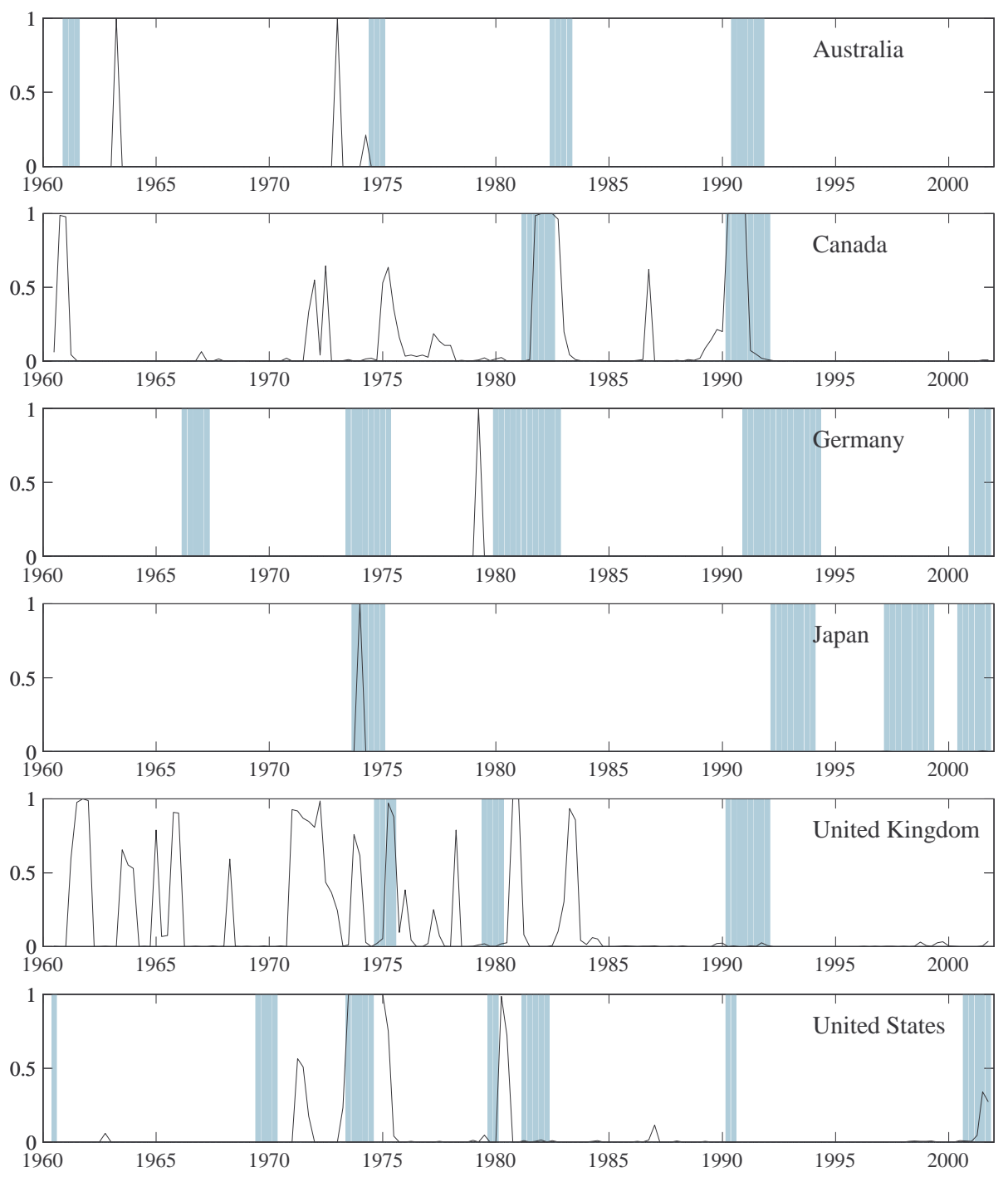

Figure 1: Posterior model probabilities of being in the low growth state, $P\left(S_{t}^{i}=1 \mid y_{T}\right)$, Markov Switching VAR. Shaded bars are recession phases from the NBER for the US, from the Melbourne Institute for Australia, and from the Economic Cycle Research Institute for all other countries. 


\subsection{Cointegration}

As discussed above, the model of Paap and Dijk (2003) is attractive for our purposes because it allows separate analysis of possible cointegration and the effects of the Markov state variables. Because the primary focus of this paper is on the latter, however, we present our cointegration results only briefly, and mainly using informal graphical methods.

We assessed the cointegrating rank of both models using the techniques described in Paap and Dijk (2003), which are based on the singular value decomposition of the $\Pi$ matrix. For both the Markov switching and linear VAR models, the posterior distributions of the cointegrating rank had mass 1 on a rank of 1 . In order to examine the cointegrating vector for each model, we imposed a rank of one and computed the posterior distribution of the $\alpha$ and $\beta$ vectors using the posterior mean cointegrating space (PMCS) estimator of Villani (2003). This estimator is attractive because it is invariant to the order of variables in the system, and does not require us to normalize on a particular coefficient. The technique is a simple modification of the procedure described in Paap and Dijk (2003), and proceeds as follows:

1. compute $\tilde{\alpha}$ and $\tilde{\beta}$ as described in Paap and Dijk (2003);

2. re-normalize $\tilde{\beta}$ to make it orthonormal: $\bar{\beta}=\tilde{\beta}\left(\tilde{\beta}^{\prime} \tilde{\beta}\right)^{-1 / 2}$ (and normalize $\tilde{\alpha}$ in a complementary manner so that the product is unchanged);

3. compute the eigenvalues of $\bar{\beta} \bar{\beta}^{\prime}$ corresponding to the $r$ largest eigenvalues (in our case, $r=1$ );

4. the PMCS estimator $\hat{\beta}$ is then the mean of $\bar{\beta} \bar{\beta}^{\prime}$ across Gibbs sampling draws.

Figures 2 and 3 show the posterior distributions of the cointegrating vector and 
corresponding $\alpha$ terms for the MS-VAR model. The only two $\beta$ coefficients with appreciable posterior mass away from zero are those corresponding to the United States and Canada. The distributions suggest that these coefficients are equal in magnitude and opposite in sign. In addition, only Canada seems to respond to movements in the error correction term. The interpretation of the cointegrating relationship is straightforward in this case, and intuitively plausible.

Things are not as clear in the linear VAR case, however. In this case it would appear from figure 4 that the $\beta$ coefficients for the United States and Germany are equal and opposite, as are those of Canada and Japan (although the latter two have appreciable mass around zero). Figure 5 suggests that all of the $\alpha$ terms are probably close to zero, with the possible exception of Japan (and to a lesser extent, Australia). Although there may be good reasons to expect cointegration between GDP in the US and Germany, it is not clear why this relationship would only "matter" to Japan. Again, there may be a plausible economic rationale for this, but we would argue that the results from the MS-VAR model have a more appealing "Occam's razor" property.

\subsection{Common Markov states}

As Paap and Dijk (2003) show, inference on whether the six countries in our sample share a common Markov state variable can be carried out in a manner analogous to that for cointegration rank, by performing a singular value decomposition on the matrix $\Gamma_{1}$. When we compute the posterior probability distribution across the possible numbers of Markov states, the inference is nearly as stark as it is in the case of cointegration. Over 96.5 per cent of the posterior probability was assigned to a model with six Markov states. There was about a 2.1 per cent probability that $\Gamma_{1}$ has a rank of five, and 1.3 per 


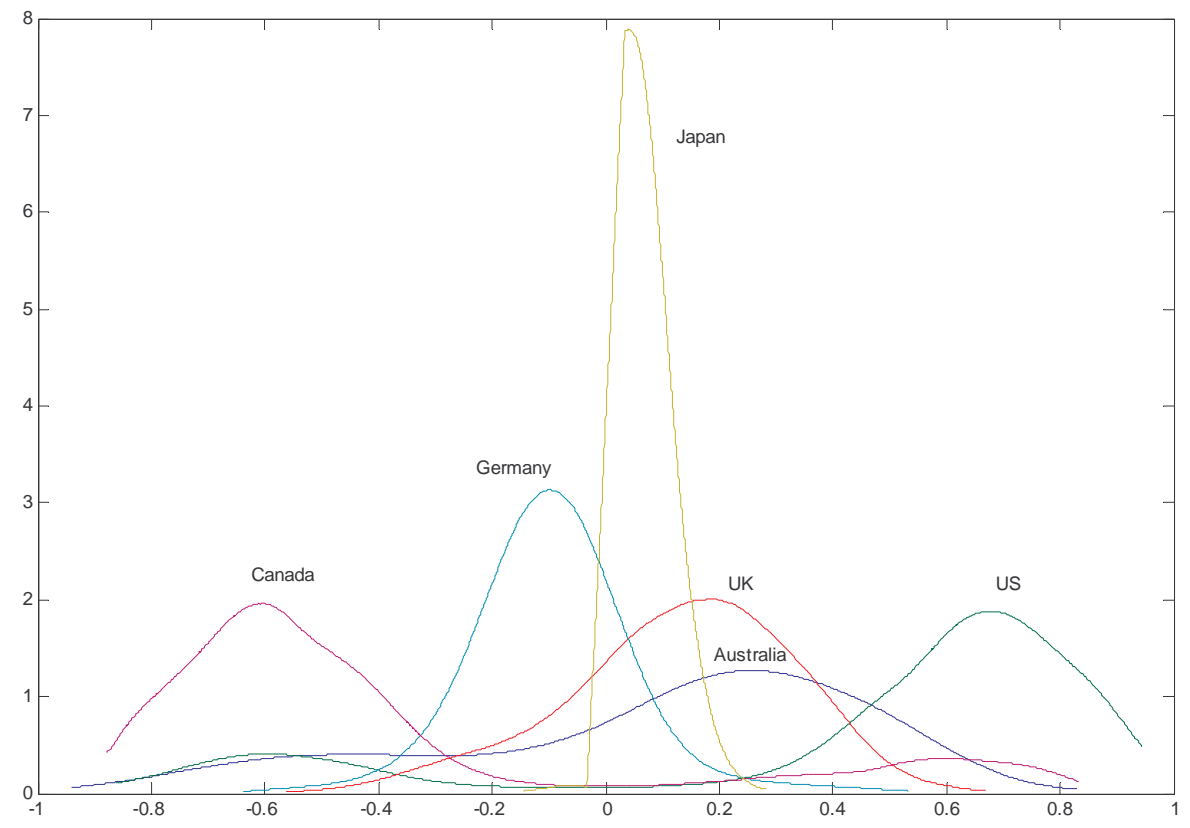

Figure 2: Posterior distributions of cointegrating vector coefficients, MS-VAR model

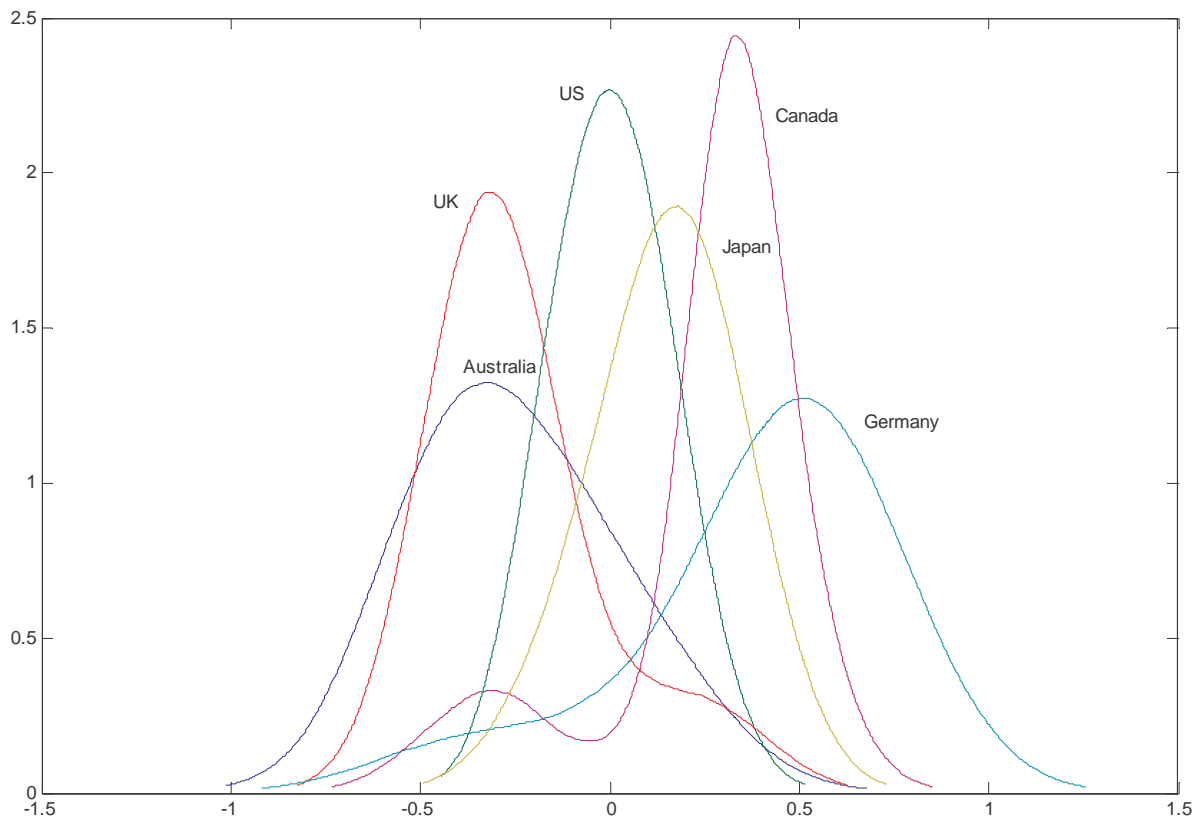

Figure 3: Posterior distributions of error-correction adjustment terms $(\alpha)$, MS-VAR 


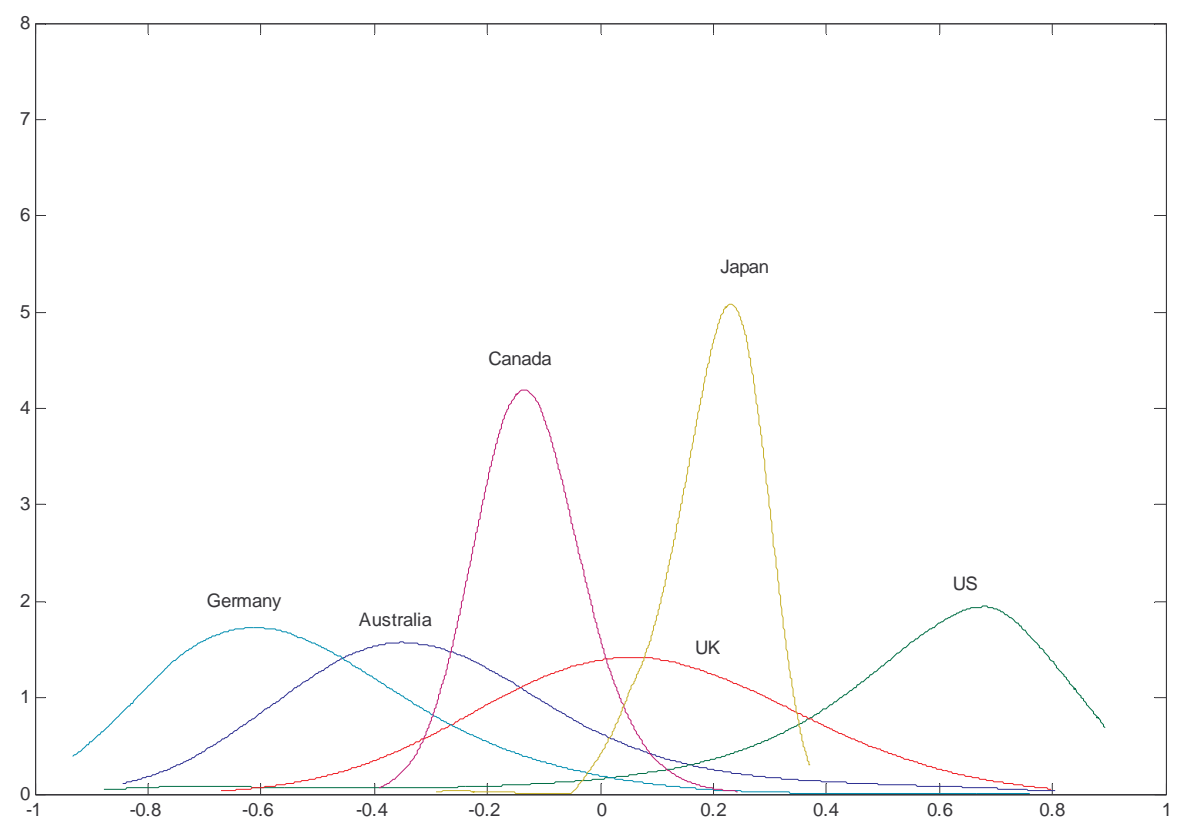

Figure 4: Posterior distributions of cointegrating vector coefficients, linear VAR model

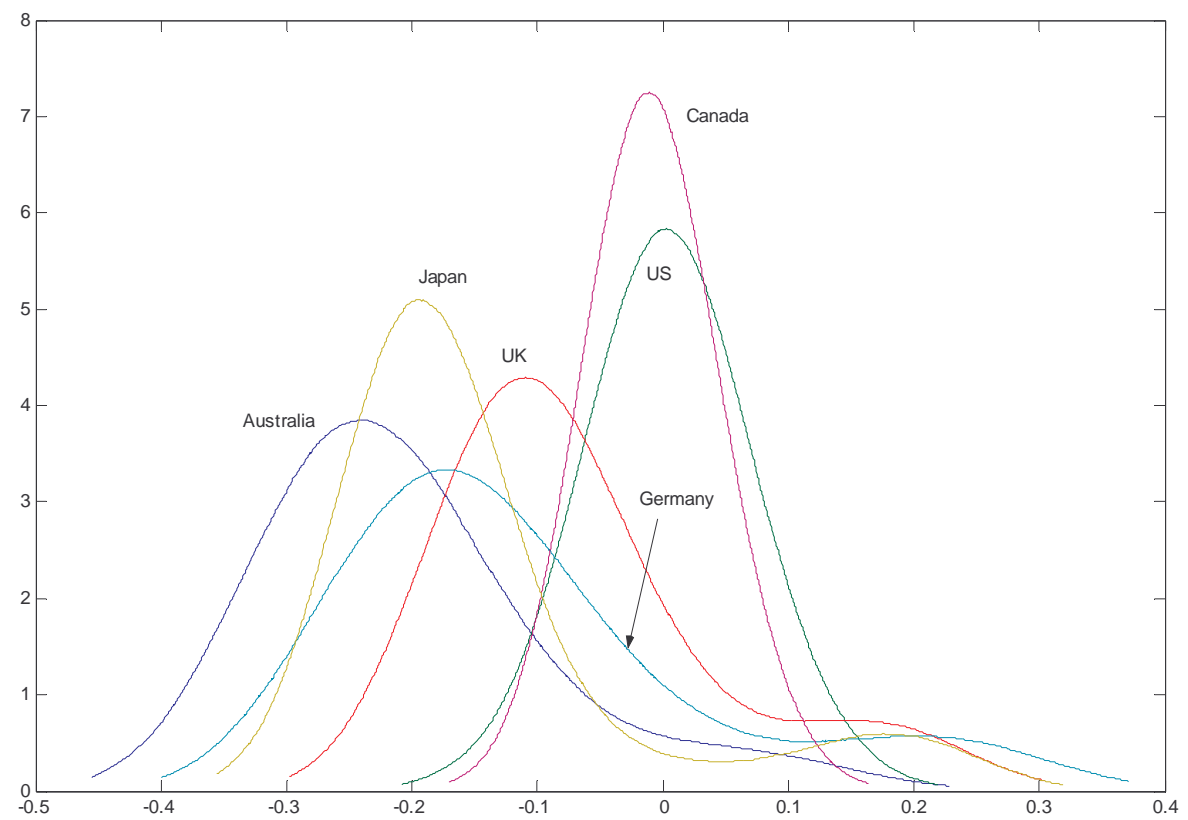

Figure 5: Posterior distributions of error-correction adjustment terms $(\alpha)$, linear VAR 
cent that it has a rank of four. Based on these results, we retain a separate Markov state variable for each country. Our results suggest that MS-VAR models such as that used by Krolzig (1997) and Artis et al. (2004), which contain a single state variable for all countries, are not supported by the data.

While these results rule out the perfect synchronization of Markov states, there is still scope for the state of one country to affect the others contemporaneously through the off-diagonal elements of $\Gamma_{1}$. This is in fact the case for a number of countries, as the parameter estimates above indicate.

\section{Posterior analysis of business cycle phases}

\subsection{Censoring of Markov states}

Once we have identified an initial set of Markov states $S_{t}$, we need to convert them into business cycle states $W_{t}$. This is necessary because we wish to assess the ability of the Markov switching model to generate "business cycles" that look like the actual ones that we observe. Our ultimate functions of interest therefore concern $W_{t}$ 's and not $S_{t}$ 's.

A common method of converting the former to the latter is to set $W_{t}=1$ if $\operatorname{Pr}\left(S_{t}=1 \mid y_{T}\right) \geq 0.5$. However, as argued by Harding and Pagan (2002), a set of turning points needs to meet several important criteria to match the definition of a business cycle as commonly used by traditional dating committees such as the NBER. These criteria include peaks and troughs that alternate and minimum durations of recessions and expansions.

The Bry and Boschan (1971) dating algorithm was developed as a transparent and automatic nonparametric method of dating the turning points of the business cycle in a monthly series. It is directly based upon the methodology of Burns and Mitchell (1946) 
and the dating committee at the NBER. Harding and Pagan (2002) adjust the algorithm for quarterly data. Given a quarterly time series, $y_{t}$ they define a trough as occurring at time $t$ whenever

$$
\left\{y_{t}-y_{t-2}<0, y_{t}-y_{t-1}<0, y_{t+1}-y_{t}>0, y_{t+1}-y_{t-1}>0\right\},
$$

ensuring that $y_{t}$ is a local minimum relative to the two quarters on either side of the observation. A peak is defined analogously as occurring whenever

$$
\left\{y_{t}-y_{t-2}>0, y_{t}-y_{t-1}>0, y_{t+1}-y_{t}<0, y_{t+1}-y_{t-1}<0\right\} .
$$

This rule, combined with some other censoring rules that impose a five quarter minimum duration for an entire cycle and a minimum of two quarters for each expansion or contraction phase, is named the BBQ algorithm by Harding and Pagan (2002). We follow Harding and Pagan and use their BBQ algorithm to map the $S_{t}$ into $W_{t}$. This procedure ensures that the simulated data from the Markov switching model is treated in the same way as the actual data, insofar as the location of turning points is concerned.

For each draw of the parameters, we constructed a data series of the same length as our observed sample, then filtered these series using the BBQ dating algorithm and NBER-style censoring rules. We have used the same procedure in earlier work (Smith and Summers (2002)) to relate Bayesian priors on model parameters to implied priors on observable features of real-world business cycles. This resulted in 75,000 simulated business cycle chronologies for each country. Because some parameter values resulted in explosive behavior in the simulated data, we impose a further "reality check." This consisted of excluding from our analysis any simulated data series in which the maximum or minimum growth rate was greater (in absolute value) than four times the observed maximum or minimum. We also exclude simulated series with less than two 
turning points. This is necessary to rule out cases in which a single expansion or contraction acts as an absorbing state. The proportion of simulated series that satisfied these criteria is given in the table below.

\begin{tabular}{lllllll}
\hline \hline Model & Australia & US & UK & Germany & Canada & Japan \\
\hline MS-VAR & 0.9962 & 0.9962 & 0.9928 & 0.9960 & 0.9941 & 0.9286 \\
VAR & 0.9803 & 0.9684 & 0.9839 & 0.9822 & 0.9241 & 0.7067 \\
\hline \hline
\end{tabular}

The "reality check" filter had more of an effect on data simulated from the linear VAR, especially for Japan. This table gives at least some informal support to the MS-VAR model, in the sense that data generated from that model are more likely to resemble our actual GDP data. $^{4}$

Figure 6 depicts the posterior probability of the business cycle states, $W_{t}$, being in a recession at each point in time. These probabilities were calculated by taking the mean of the 75,000 draws of the business cycle state vector at each time $t$ from the Markov switching VAR. A similar picture for the linear VAR is presented in figure 7.

The contrast between the performance of the two models highlighted by figures 6 and 7 is striking. It is not correct to infer from figure 7 that the linear VAR model does not generate turning points or cycles. Indeed, we demonstrate below that the linear VAR does in fact generate turning points - see table 2 and figures 8 and 10 - and that in many cases this model does as well as the MS-VAR when compared to the data. The message from figure 7 is that these cycles occur with roughly equal probability at all dates throughout the sample period, and so get averaged out across Gibbs draws. Furthermore, because the residual covariance matrix is not diagonal, the contemporaneous correlation of shocks across countries means that the cycles generated by the VAR tend

\footnotetext{
${ }^{4}$ It is important to note that we are not simulating data in the same way as, for example, Harding and Pagan (2002). An equivalent procedure in our case would involve generating a large number of simulated data series with the parameter values fixed at their posterior means. Here we are interested in the nature of the posterior distributions of quantities such as $p\left(W_{t}^{U S}=1\right)$, or the correlation between $W_{t}^{U S}$ and $W_{t}^{C A}$. It is these distributions that we are really "simulating."
} 
to have concordance and correlation values that are not too dissimilar to the observed data (we discuss these measures below). In other words, given any single draw of the parameters, turning points may occur near each other. But there is no reason to expect them to occur at same points in the sample across draws.

On the other hand, the Markov switching VAR has additional information about where turning points are likely to be located over the sample period. Although the unobserved Markov states $S_{t}$ are different from the business cycle states $W_{t}$, the former do seem to convey important information about the temporal location of the latter. ${ }^{5}$

\subsection{Assessing synchronization}

Recent papers by Artis et al. (1997) and Bodman and Crosby (2000) employ nonparametric methods to test for the independence of business cycles in the G7 countries (the United States, Japan, Germany, the United Kingdom, France, Italy, and Canada). Both papers rely (implicitly or explicitly) on a binary indicator variable, taking the value one in expansions and zero in recessions. The indicator variable is constructed from a business cycle chronology for each country. ${ }^{6}$ Artis et al. (1997) use a version of the Bry-Boschan algorithm to obtain their chronologies. Once the peak and trough dates (i.e., the beginning dates of recessions and expansions, respectively) have been identified for each country, these authors use Pearson's contingency coefficient to test the null hypothesis of independence of the G7 business cycles.

Alternatively, Bodman and Crosby (2000) treat the product $\left(1-W_{x t}\right)\left(1-W_{y t}\right)$ as an independent Bernoulli random variable for each country. These authors are thus specifically testing the independence of recessions in each country.

\footnotetext{
${ }^{5}$ This information is, of course, dependent on the entire observed sample. If we were forecasting future business cycles, for instance, then as the forecast horizon lengthens, the probability of future recessions from the MS-VAR model would converge to the unconditional mean (but calculated from the $W^{\prime} s$, not the $S^{\prime} s$ ).

${ }^{6}$ In studies that employ Markov switching models, $W_{t}$ is typically derived from the state variable $S_{t}$ described above, by a rule such as $W_{t}=1$ if $\operatorname{Pr}\left(S_{t}=0 \mid y_{T}\right)>0.5$ (recall that our parameterization identifies $S_{t}=0$ with periods of high growth).
} 

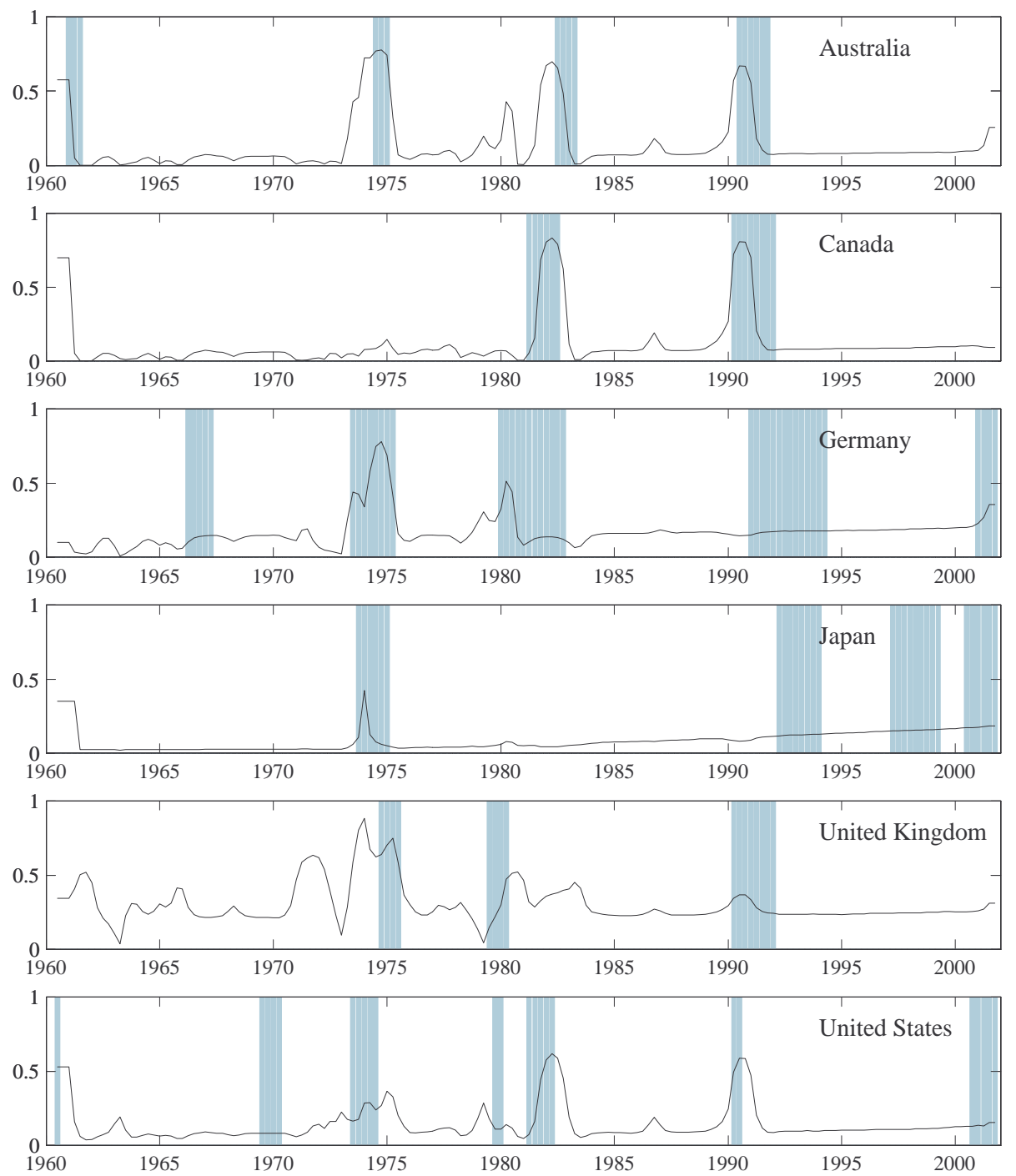

Figure 6: Posterior model probabilities of being in recession, $P\left(W_{t}=1\right)$, Markov Switching VAR 

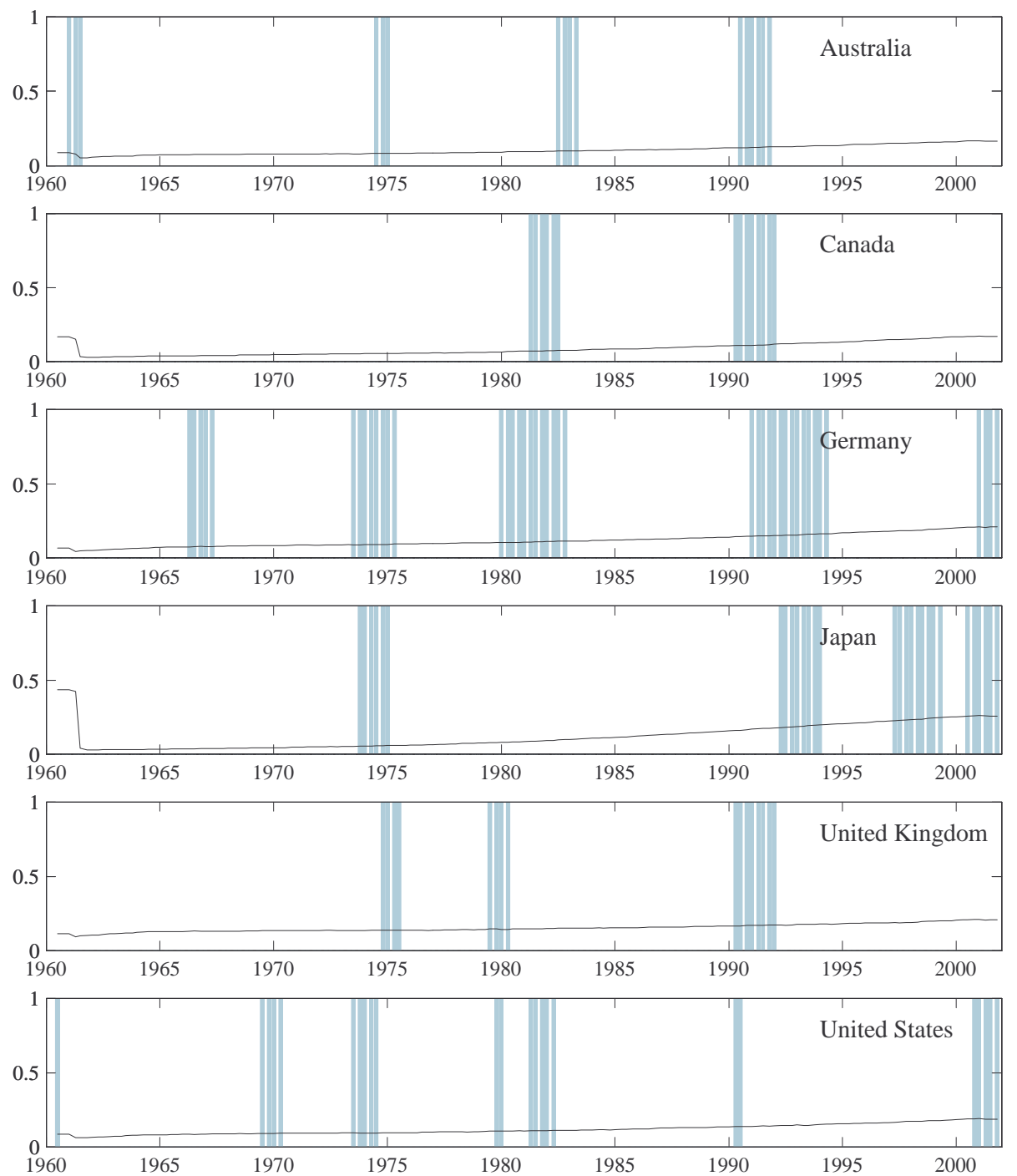

Figure 7: Posterior model probabilities of being in recession, $P\left(W_{t}=1\right)$, linear VAR 
A third measure of synchronization of two or more business cycles is the concordance index described in Harding and Pagan (2003) . They define the concordance index, $\hat{I}$, between two binary series as the average fraction of the sample for which the two series are in the same state:

$$
\hat{I}=T^{-1}\left[\sum_{t=1}^{T} W_{x t} W_{y t}+\sum_{t=1}^{T}\left(1-W_{x t}\right)\left(1-W_{y t}\right)\right],
$$

where $T$ is the sample size. Harding and Pagan note that this can be re-written as

$$
\hat{I}=1+2\left(\hat{\rho}_{W} \hat{\sigma}_{W x} \hat{\sigma}_{W y}+\hat{\mu}_{W x} \hat{\mu}_{W y}\right)-\hat{\mu}_{W x}-\hat{\mu}_{W y} .
$$

In this expression, $\hat{\mu}_{W x}$ and $\hat{\sigma}_{W x}$ are the estimated mean and standard deviation of $W_{x}$, etc., while $\hat{\rho}_{W}$ is the correlation between $W_{x}$ and $W_{y}$. As Harding and Pagan point out, the usefulness of equation (9) is that it makes clear that the value of $\hat{I}$ depends on both the correlation between the two series and on how often they are in the expansion state $W=1$. In measuring synchronization between cycles in industrial production for several countries, Harding and Pagan note that it is often the case that a high value of $\hat{I}$ coincides with a low value of $\hat{\rho}$ (for a given pair of series). We find a similar phenomenon when we examine cycles in GDP across the six countries listed above. For most of the country pairs that we examine, the posterior distribution of $\hat{I}$ implied by the MS-VAR model is a much better description of the actual data than is the posterior of $\hat{\rho}$.

In the next two sub-sections, we assess the extent to which the United States business cycle is synchronized with those of the other countries, by presenting the posterior distributions of both $\hat{I}$ and $\rho$. We constructed the prior and posterior distributions of the concordance and correlation measures using the corresponding distribution of the parameters of the MS-VAR and linear VAR models, via the simulated data series de- 
scribed above.

\subsection{Posterior distributions of the concordance statistics}

The results of our analysis of concordance among business cycles in the six countries are shown below the diagonal in tables 1 and 2 and also in figure 8 . In each figure, we present the posterior distributions of the concordance statistics generated by the MSVAR (dotted lines) and the VAR model (unbroken lines), along with the actual value computed from the business cycle chronology for each country (the vertical dashed lines). ${ }^{7}$ A comparison of the prior and posterior distributions, as implied by the prior distribution on the linear VAR and MS-VAR parameters, is presented in figure 9 for the United States and Canada. The priors are similar for the other country pairs.

For all the country pairs, the prior distribution of the concordance index is quite diffuse relative to the posterior. The flatness of the prior implies a belief that the business cycle states across countries are just as likely to be out of phase as they are to be in phase. The data are reasonably informative about the amount of time any given pair of countries could be expected to be in the same business cycle state. Furthermore, the mass of the posterior distributions of the concordance statistics implied by the MSVAR is closer to the value of the data for 10 out of the 15 country pairs. This suggests that the underlying MS-VAR model provides a slightly better framework for studying this aspect of synchronization than the linear VAR.

\footnotetext{
${ }^{7}$ To compute the actual values, we use the NBER business cycle chronology for the United States (http://www.nber.org/cycles/), and the Melbourne Institute's chronology for Australia (http://wff2.ecom.unimelb.edu.au/iaesrwww/bcf/bdates5197.html). All other chronologies are from the Economic Cycle Research Institute (ECRI; http://www.businesscycle.com/research/intlcycledates.php).
} 

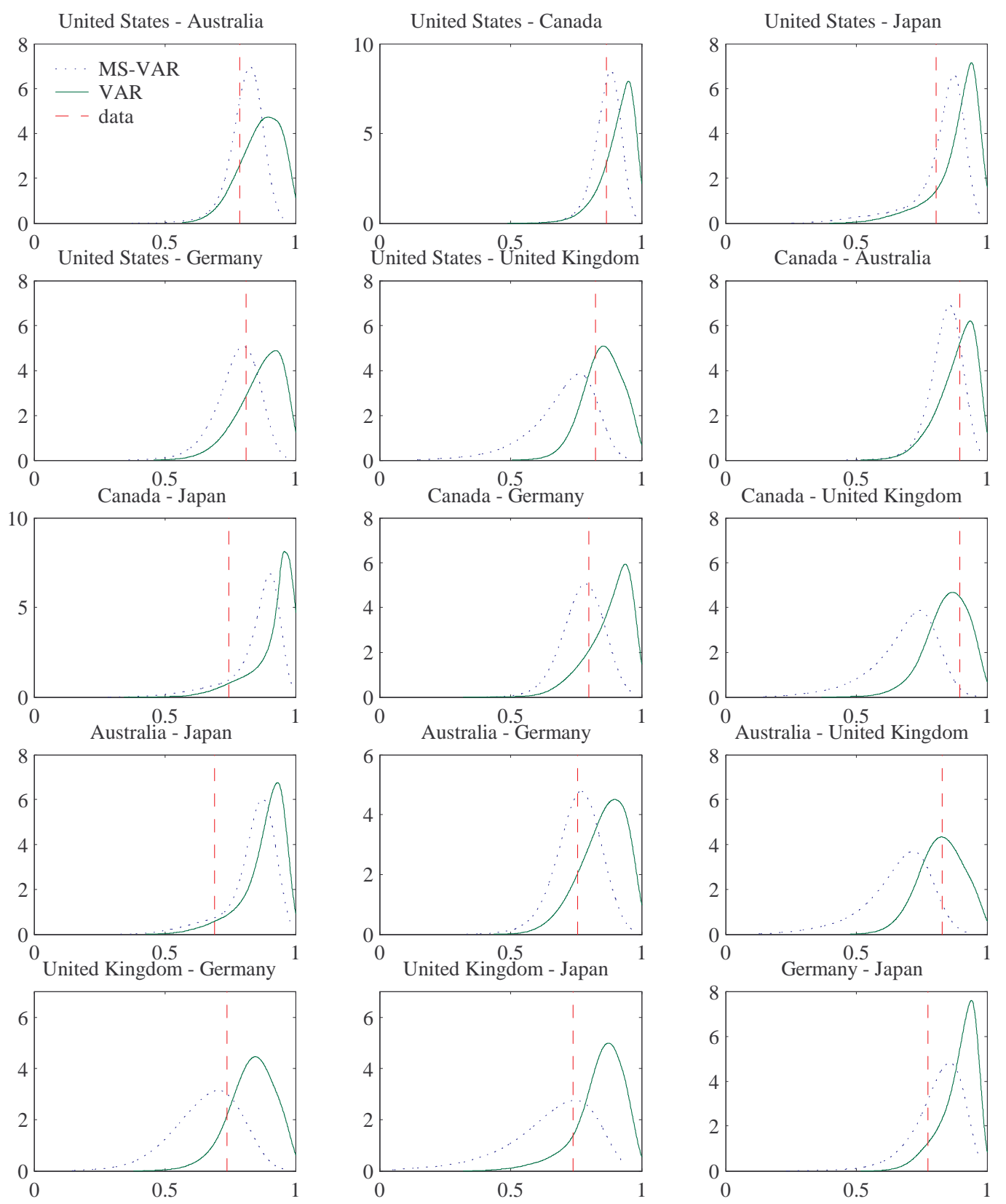

Figure 8: Posterior distribution of concordance indexes 
Table 1: Posterior quantiles of business cycle concordances, MS-VAR model

\begin{tabular}{|c|c|c|c|c|c|}
\hline & Australia & Canada & Japan & Germany & United Kingdom \\
\hline \multirow[t]{4}{*}{ United States } & $0.8085^{\mathrm{a}}$ & 0.8662 & 0.8185 & 0.7749 & 0.6980 \\
\hline & $(0.0682)$ & $(0.0552)$ & $(0.1116)$ & $(0.0845)$ & $(0.1251)$ \\
\hline & [0.8310] & {$[0.8873]$} & [0.8803] & {$[0.8010]$} & {$[0.7726]$} \\
\hline & 0.7866 & 08659 & 0.8049 & 0.8110 & 0.8232 \\
\hline \multirow[t]{4}{*}{ Australia } & & 0.8412 & 0.8311 & 0.7566 & 0.6575 \\
\hline & & $(0.0642)$ & $(0.0990)$ & $(0.0876)$ & $(0.1282)$ \\
\hline & & {$[0.8624]$} & {$[0.8756]$} & {$[0.7640]$} & {$[0.7211]$} \\
\hline & & 0.8963 & 0.6890 & 0.7561 & 0.8293 \\
\hline \multirow[t]{4}{*}{ Canada } & & & 0.8490 & 0.7742 & 0.6816 \\
\hline & & & $(0.1036)$ & $(0.0812)$ & $(0.1274)$ \\
\hline & & & {$[0.9085]$} & {$[0.7895]$} & {$[0.7528]$} \\
\hline & & & 0.7439 & 0.7988 & 0.8963 \\
\hline \multirow[t]{4}{*}{ Japan } & & & & 0.8097 & 0.6557 \\
\hline & & & & $(0.0995)$ & $(0.1627)$ \\
\hline & & & & {$[0.8716]$} & {$[0.7596]$} \\
\hline & & & & 0.7744 & 0.7378 \\
\hline \multirow[t]{4}{*}{ Germany } & & & & & 0.6577 \\
\hline & & & & & $(0.1282)$ \\
\hline & & & & & {$[0.7030]$} \\
\hline & & & & & 0.7378 \\
\hline
\end{tabular}

${ }^{\text {a }}$ For each country pair, the rows in the table show the posterior mean, (standard deviation), [mode], and data value

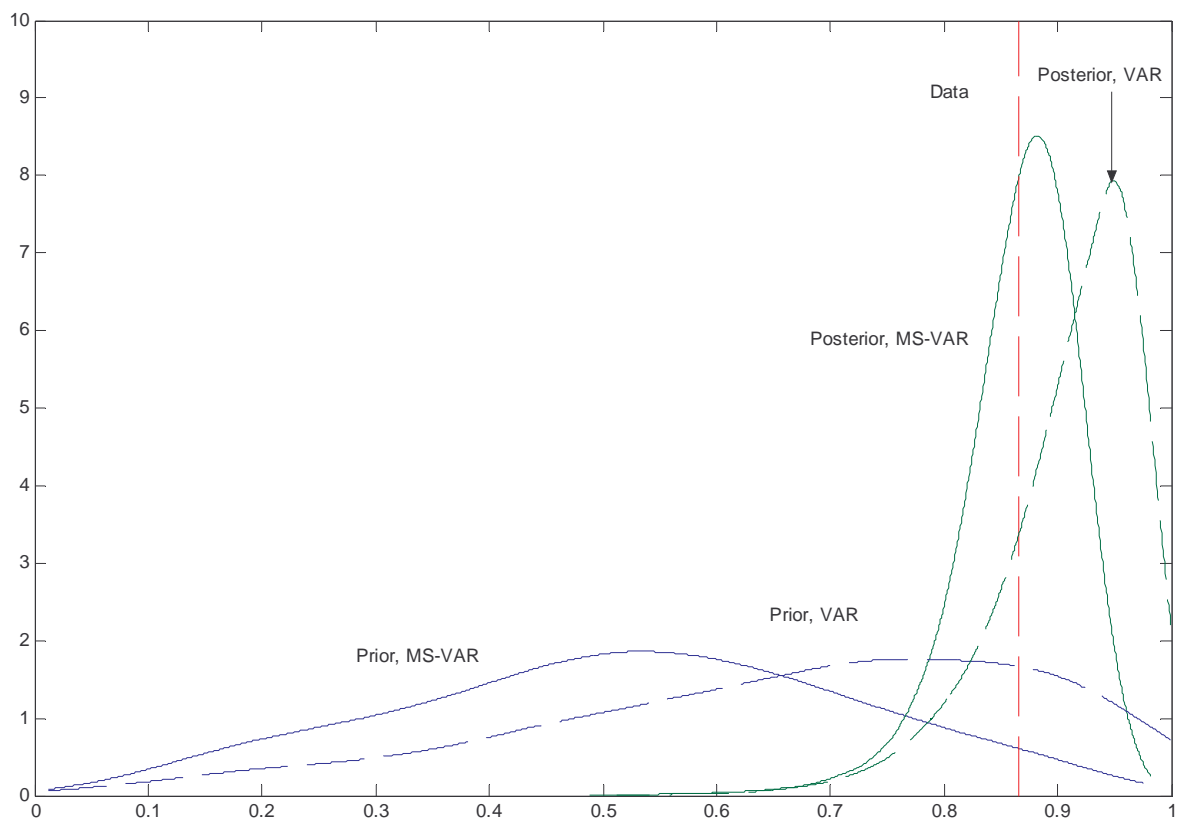

Figure 9: Prior and posterior distributions of business cycle concordance between the US and Canada 
Table 2: Posterior quantiles of business cycle concordances, linear VAR model

\begin{tabular}{|c|c|c|c|c|c|}
\hline & Australia & Canada & Japan & Germany & United Kingdom \\
\hline \multirow{4}{*}{ United States } & $0.8644^{\mathrm{a}}$ & 0.9104 & 0.8855 & 0.8642 & 0.8509 \\
\hline & $(0.0795)$ & $(0.0667)$ & $(0.0940)$ & $(0.0871)$ & $(0.0756)$ \\
\hline & {$[0.8958]$} & {$[0.9631]$} & {$[0.9531]$} & {$[0.9441]$} & {$[0.8464]$} \\
\hline & 0.7866 & 0.8659 & 0.8049 & 0.8110 & 0.8232 \\
\hline \multirow[t]{4}{*}{ Australia } & & 0.8846 & 0.8783 & 0.8517 & 0.8242 \\
\hline & & $(0.0746)$ & $(0.0873)$ & $(0.0894)$ & $(0.0872)$ \\
\hline & & [0.9461] & {$[0.9376]$} & [0.8972] & [0.8323] \\
\hline & & 0.8963 & 0.6890 & 0.7561 & 0.8293 \\
\hline \multirow{4}{*}{ Canada } & & & 0.9137 & 0.8769 & 0.8453 \\
\hline & & & $(0.0919)$ & $(0.0891)$ & $(0.0871)$ \\
\hline & & & {$[0.9636]$} & {$[0.9573]$} & {$[0.8696]$} \\
\hline & & & 0.7439 & 0.7988 & 0.8963 \\
\hline \multirow[t]{4}{*}{ Japan } & & & & 0.8930 & 0.8405 \\
\hline & & & & $(0.0711)$ & $(0.0974)$ \\
\hline & & & & {$[0.9509]$} & {$[0.8760]$} \\
\hline & & & & 0.7744 & 0.7378 \\
\hline \multirow[t]{4}{*}{ Germany } & & & & & 0.8303 \\
\hline & & & & & $(0.0902)$ \\
\hline & & & & & {$[0.8533]$} \\
\hline & & & & & 0.7378 \\
\hline
\end{tabular}

${ }^{\text {a }}$ For each country pair, the rows in the table show the posterior mean, (standard deviation), [mode], and data value

\subsection{Posterior distributions of the correlation statistics}

Posterior distributions of the bilateral correlations of censored business cycle states for the MS-VAR and VAR are presented in tables 3 and 4 and in figure 10. Again, the dotted line depicts the posterior distribution of the concordance statistic for the MS-VAR, while the solid line depicts the posterior distribution of the concordance statistic for the VAR. As with the figures displaying the concordance statistics, the simulated prior distributions and actual data values (the vertical broken lines) for these correlations have also been plotted, for the US and Canada, in figure 11. 

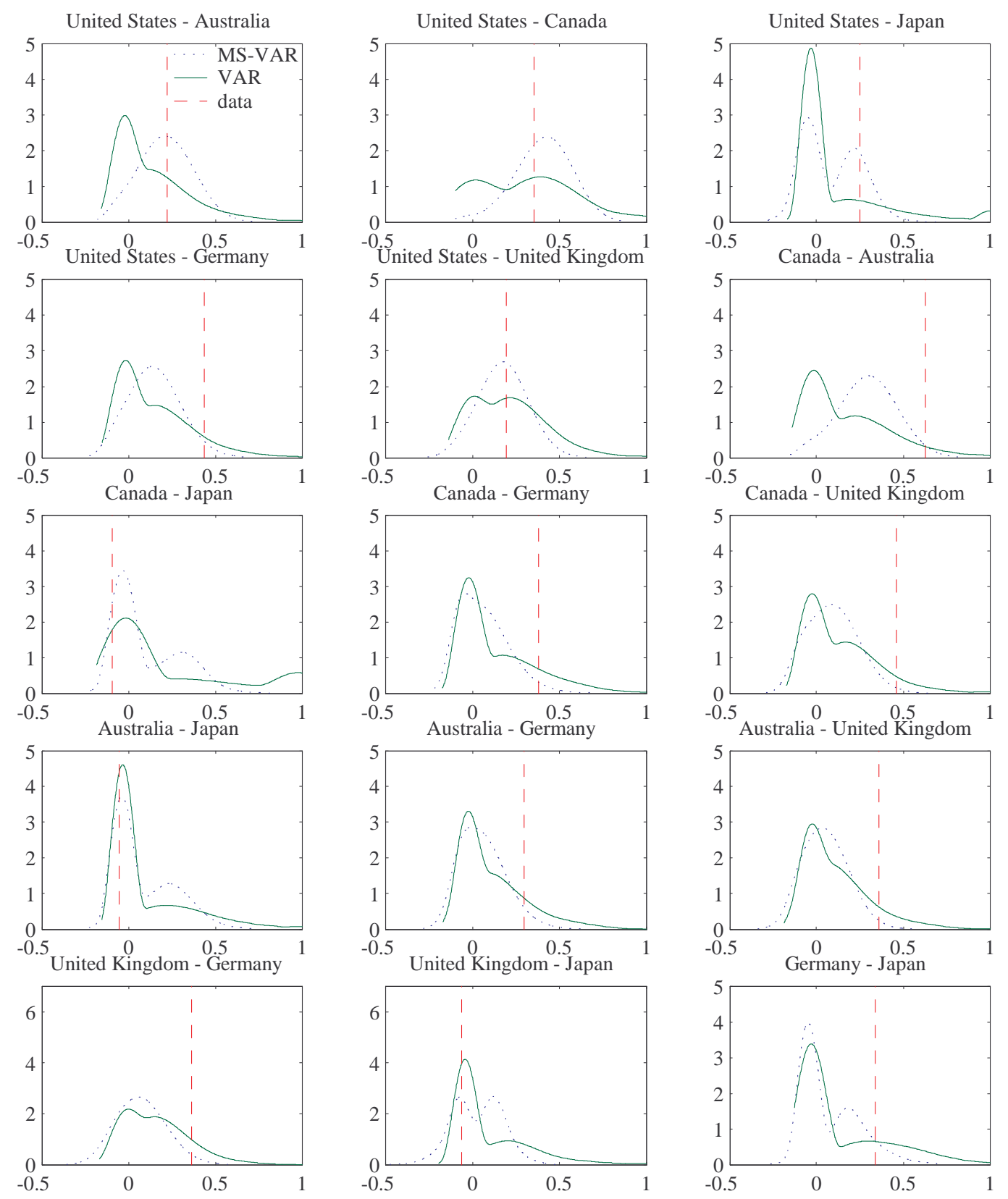

Figure 10: Posterior distribution of correlations 
Table 3: Posterior quantiles of business cycle correlations, MS-VAR model

\begin{tabular}{llllll}
\hline \hline & Australia & Canada & Japan & Germany & United Kingdom \\
\hline United States & $0.2115^{\mathrm{a}}$ & 0.4026 & 0.0880 & 0.1478 & 0.1738 \\
& $(0.1562)$ & $(0.1674)$ & $(0.1631)$ & $(0.1508)$ & $(0.1444)$ \\
& {$[0.2078]$} & {$[0.4428]$} & {$[-0.0385]$} & {$[0.1427]$} & {$[0.1840]$} \\
& 0.2212 & 0.3530 & 0.2493 & 0.4338 & 0.1929 \\
Australia & & 0.2914 & 0.0875 & 0.0584 & 0.0512 \\
& & $(0.1693)$ & $(0.1741)$ & $(0.1374)$ & $(0.1338)$ \\
& & {$[0.3210]$} & {$[-0.0327]$} & {$[-0.0297]$} & {$[0.0363]$} \\
Canada & 0.6286 & -0.0535 & 0.2967 & 0.3605 \\
& & & 0.1009 & 0.0622 & 0.0916 \\
& & & $(0.1903)$ & $(0.1461)$ & $(0.1485)$ \\
Japan & & $-0.0299]$ & {$[-0.0610]$} & {$[0.0936]$} \\
& & & -0.0946 & 0.3797 & 0.4589 \\
& & & 0.0744 & 0.0286 \\
Germany & & & $(0.1661)$ & $(0.1435)$ \\
& & & & {$[-0.0402]$} & {$[-0.0593]$} \\
& & & & -0.0630 \\
& & & & 0.0708 \\
& & & & $0.1452)$ \\
\hline \hline
\end{tabular}

${ }^{a}$ For each country pair, the rows in the table show the posterior mean, (standard deviation), [mode], and data value

The striking feature of these distributions is that the posterior distributions of the correlation statistics are bimodal for nearly all of the country pairs for the VAR and also for several of the country pairs for the MS-VAR. This is related to the relatively informative prior on the correlation statistics implied by the prior of the VAR and MSVAR models. As the data is simulated under the prior of a linear model with diagonal covariance and null correlation matrices, a large mass of the prior distribution of the correlation statistic is concentrated around zero. This is in stark contrast to the relatively flat distributions of the prior on the concordance indexes depicted in figure 9. If the data are not very informative about the off-diagonal elements of the $\Gamma_{1}, \bar{\Phi}, \Pi$ or $\Sigma$ matrices, then there will be very little clustering of turning points and the posterior distribution of the correlations between the business cycle states of country pairs will 
Table 4: Posterior quantiles of business cycle correlations, linear VAR model

\begin{tabular}{|c|c|c|c|c|c|}
\hline & Australia & Canada & $\overline{\text { Japan }}$ & Germany & United Kingdom \\
\hline \multirow[t]{4}{*}{ United States } & $0.1362^{\mathrm{a}}$ & 0.3140 & 0.1227 & 0.1464 & 0.2113 \\
\hline & $(0.2156)$ & $(0.2824)$ & $(0.2753)$ & $(0.2078)$ & $(0.2137)$ \\
\hline & {$[-0.0279]$} & {$[-0.0198]$} & {$[-0.0327]$} & {$[-0.0221]$} & {$[-0.0281]$} \\
\hline & 0.2212 & 0.3530 & 0.2493 & 0.4338 & 0.1929 \\
\hline \multirow[t]{4}{*}{ Australia } & & 0.1763 & 0.1076 & 0.1149 & 0.1146 \\
\hline & & $(0.2403)$ & $(0.2377)$ & $(0.1956)$ & $(0.1931)$ \\
\hline & & {$[-0.0229]$} & {$[-0.0266]$} & {$[-0.0293]$} & {$[-0.0315]$} \\
\hline & & 0.6286 & -0.0535 & 0.2967 & 0.3605 \\
\hline \multirow[t]{4}{*}{ Canada } & & & 0.2522 & 0.1374 & 0.1392 \\
\hline & & & $(0.3885)$ & $(0.2253)$ & $(0.2112)$ \\
\hline & & & {$[-0.0188]$} & {$[-0.0261]$} & {$[-0.0278]$} \\
\hline & & & -0.0946 & 0.3797 & 0.4589 \\
\hline \multirow[t]{4}{*}{ Japan } & & & & 0.1410 & 0.0937 \\
\hline & & & & $(0.2581)$ & $(0.2166)$ \\
\hline & & & & {$[-0.0251]$} & {$[-0.0444]$} \\
\hline & & & & 0.3368 & -0.0630 \\
\hline \multirow[t]{4}{*}{ Germany } & & & & & 0.1592 \\
\hline & & & & & $(0.1880)$ \\
\hline & & & & & {$[-0.0239]$} \\
\hline & & & & & 0.3630 \\
\hline
\end{tabular}

${ }^{\text {a }}$ For each country pair, the rows in the table show the posterior mean, (standard deviation), [mode], and data value

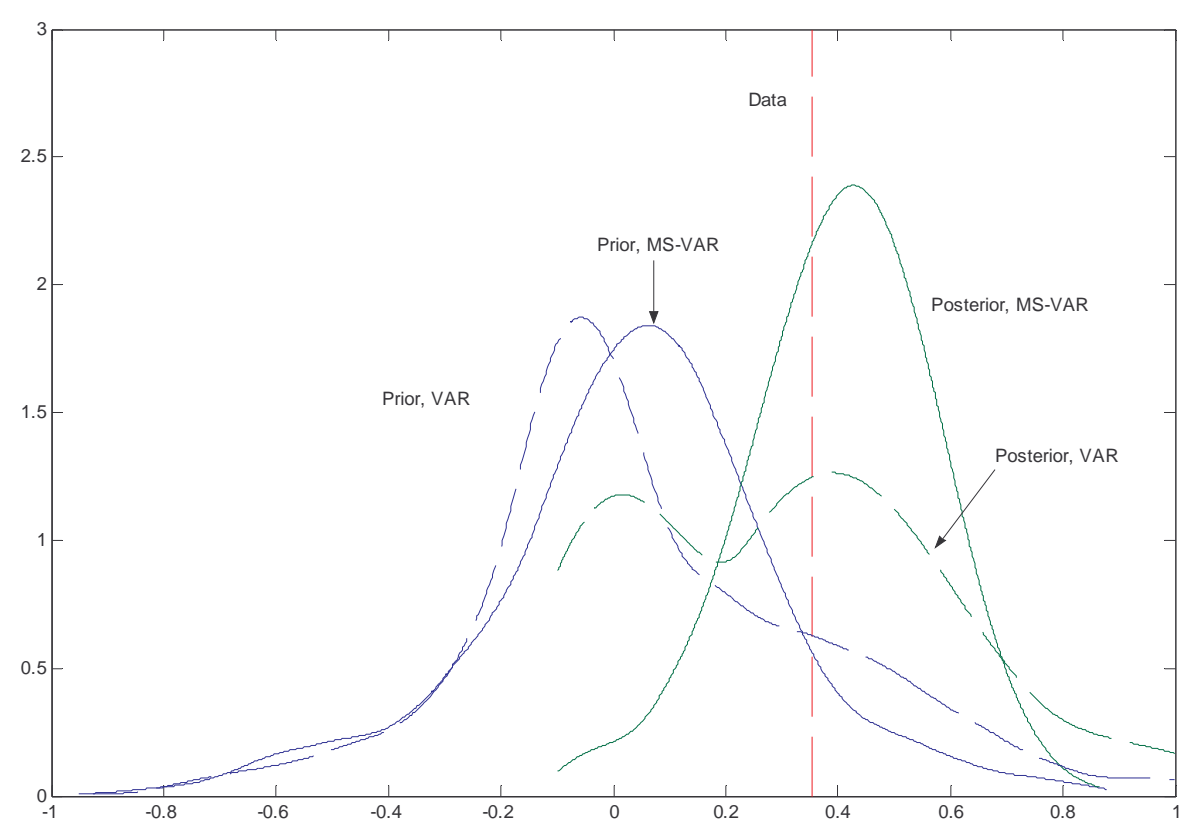

Figure 11: Prior and posterior distributions of business cycle correlation between the US and Canada 
also have considerable mass around zero.

The MS-VAR is noticeably more informative about the nature of correlations between business cycle states across countries than the linear VAR model, and usually better at capturing the actual correlations. This is because the MS-VAR provides additional information about the temporal location of turning points. In addition, the mass of the posterior distribution of the correlation statistics for the MS-VAR lies closer to the correlation for the data than does the linear VAR for most models. However, while the MS-VAR is more informative than the VAR about the correlation between business cycle states, the only country pair for which the MS-VAR exhibits significant correlation (i.e., a t-ratio of at least 2) is the United States and Canada.

Harding and Pagan (2003, p. 14 and 25) present correlation statistics and associated robust t-ratios for industrial production in several OECD countries. They also find quite small correlations relative to the estimated concordance statistics, although the correlations are generally somewhat larger in absolute value than the modes of our posterior distributions. For the country pairs in common with our sample, they find only the correlations between the Unites States and Canada and Japan and Germany to be significant at the $95 \%$ level. Given that the MS-VAR performs quite poorly in locating business cycle turning points in our GDP data for both Germany and Japan, it is not surprising that the MS-VAR fails to capture much correlation between these two countries.

\section{Conclusions and future directions}

This paper has analyzed the extent of synchronization in fluctuations of real GDP across several industrialized countries using a Markov switching VAR. We find little evidence of synchronization in the sense of either a common Markov trend or covari- 
ance of country specific impulses across countries. However, we do find evidence of a long-run cointegrating relationship between the United States and Canada. Simulating data from the posterior distribution of the MS-VAR, we find that the only country pair for which the mass of the posterior distribution of the correlation between business cycle turning points lies away from zero is the United States and Canada.

In the title, we ask how well Markov switching models perform in measuring synchronization. We find that the MS-VAR model appears to perform quite well in capturing the synchronization of classical business cycles observed in standard reference chronologies and certainly performs better than the linear VAR. This is because business cycles simulated from a linear VAR occur with roughly equal probability throughout the sample, whereas MS-VAR models provide additional information about the location of turning points in the data. This result has implications for real business cycle researchers who use VAR models to measure co-movements in the data. Because dynamic, stochastic general equilibrium models rely on VAR representations of a (log) linearized version of a model, the "co-movement" they produce will not necessarily resemble the temporal aspects of actual business cycles. In many cases, these temporal aspects are not of primary interest. However, they are essential for answering questions such as whether business cycles have become more or less synchronized in recent years.

There are several issues that remain unresolved. The first is that we have simulated data from the entire posterior distributions of the models' parameters. It is possible that both models may have performed better in reproducing observed correlations and concordances if we had instead simulated the data from a high-probability region of the posterior distribution such as the posterior mean or mode. Secondly, it may be informative to allow for a richer dependence on lags of Markov states. This could 
be achieved by including $S_{t-1}^{U S}$ in the determination of $S_{t}^{C A}$ or allowing for duration dependence along the lines of Filardo and Gordon (1998).

Finally, McConnell and Perez-Quiros (2000), Kim and Nelson (1999a) and Stock and Watson (2002) amongst others, document a reduction in the volatility of output growth for the US and several other countries since the early 1980's. In addition, Kose et al. (2003) document an increase in the importance of a global factor in explaining output variances in the G-7 since 1986, while Bordo and Helbling (2003) find evidence that business cycles have become steadily more synchronized over the course of twentieth century. For a given covariance, a reduction in the idiosyncratic variances will lead to an increase in the correlation between two series. It would therefore be useful to test whether or not any apparent increase in synchronization is a product of increased covariance or decreased country-specific variances. One way of doing this would be to specify the covariance matrix as following an independent Markov process. This is a multivariate generalization of McConnell and Perez-Quiros (2000), Kim and Nelson (1999a) and Smith and Summers (2002). However, such a generalization would also imply allowing the value of the Markov variable governing the covariance matrix to affect the mean growth rates, and vice versa. There is also the issue of whether to allow for switching in the covariances between countries. We choose to leave the investigation of these issues to future work.

\section{References}

Albert, J. H. and Chib, S.: 1993, Bayes inference via Gibbs sampling of autoregressive time series subject to markov mean and variance shifts, Journal of Business and Economic Statistics 11(1), 1-15.

Artis, M. J., Kontolemis, Z. G. and Osborne, D. R.: 1997, Business cycles for G7 and 
European countries, Journal of Business 70(2), 249-279.

Artis, M., Krolzig, H.-M. and Toro, J.: 2004, The European business cycle, Oxford Economic Papers 56, 1-44.

Bodman, P. and Crosby, M.: 2000, Are international business cycles independent?, mimeo, The University of Melbourne .

Bordo, M. D. and Helbling, T.: 2003, Have national business cycles become more synchronized?, Working Paper w10130, NBER.

Bry, G. and Boschan, C.: 1971, Cyclical analysis of time series: Selected procedures and computer programs, National Bureau of Economic Research Technical paper no. 20 .

Burns, A. F. and Mitchell, W. C.: 1946, Measuring Business Cycles, National Bureau of Economic Research, New York.

Durland, J. M. and McCurdy, T. H.: 1994, Duration-dependent transitions in a Markov model of U.S. GNP growth, Journal of Business and Economic Statistics 12(3), 279-288

Filardo, A. J.: 1994, Business cycle phases and their transitional dynamics, Journal of Business and Economic Statistics 12(3), 299-308.

Filardo, A. J. and Gordon, S. F.: 1998, Business cycle durations, Journal of Econometrics $\mathbf{8 5}, 99-123$.

Geweke, J.: 1991, Efficient simulation from the multivariate normal and student-t distributions subject to linear constraints, in E. M. Keramidas (ed.), Computing Science and Statistics: Proceedings of the Twenty-Third Symposium on the Interface, American Statistical Association, pp. 571-578. 
Goodwin, T. H.: 1993, Business-cycle analysis with a Markov-switching model, Journal of Business and Economic Statistics 11, 331-339.

Guha, D. and Banerji, A.: 1998, Testing for cycles: A Markov switching approach, Economic Cycle Research Institute Working Paper .

Hajivassiliou, V. A. and McFadden, D. L.: 1998, The method of simulated scores for the estimation of LDV models, Econometrica 66(4).

Hajivassiliou, V., McFadden, D. and Ruud, P.: 1996, Simulation of multivariate normal rectangle probabilities and their derivatives theoretical and computational results, Journal of Econometrics 72(1-2), 85-134.

Hamilton, J. D.: 1989, A new approach to the economic analysis of non-stationary time series and the business cycle, Econometrica 57, 357-384.

Hamilton, J., Waggoner, D. F. and Zha, T.: 2003, Normalization in econometrics.

Harding, D. and Pagan, A.: 2003, Synchronisation of cycles, mimeo, Department of Economics, The University of Melbourne.

Harding, D. and Pagan, A. R.: 2002, Dissecting the cycle: A methodological investigation, Journal of Monetary Economics 49(2), 365-381.

Hess, G. D. and Iwata, S.: 1997, Measuring and comparing business-cycle features, Journal of Business and Economic Statistics 15(4), 432-444.

Kass, R. E. and Raftery, A. E.: 1995, Bayes factors, Journal of the American Statistical Association 90(430), 773-795.

Keane, M.: 1990, Four Essays in Empirical Macro and Labor Economics, PhD thesis, Brown University. 
Kim, C.-J. and Nelson, C. R.: 1999a, Has the U.S. economy become more stable? a Bayesian approach based on a Markov-switching model of business cycle, Review of Economics and Statistics 81(4), 608-616.

Kim, C.-J. and Nelson, C. R.: 1999b, State-Space Models with Regime Switching: Classical and Gibbs-Sampling Approaches with Applications, MIT Press, Cambridge, MA.

Kose, M. A., Otrok, C. and Whiteman, C. H.: 2003, International business cycles: World, region, and country-specific factors, American Economic Review 93(4), 1216-1239.

Krolzig, H.-M.: 1997, Markov-Switching Vector Autoregressions: Modelling, Statistical Inference, and Application to Business Cycle Analysis, Springer-Verlag, Berlin.

Lam, P.-S.: 1990, The Hamilton model with a general autoregressive component: Estimation and comparison with other models of economic time series, Journal of Monetary Economics 26, 409-432.

McConnell, M. M. and Perez-Quiros, G.: 2000, Output fluctuations in the United States: What has changed since the early 1980's?, American Economic Review 90(5), 1464-1476.

McCulloch, R. E. and Tsay, R. S.: 1994, Statistical analysis of economic time series via Markov switching models, Journal of Time Series Analysis 15(5), 523-538.

Newton, M. A. and Raftery, A. E.: 1994, Approximate Bayesian inference by the weighted likelihood bootstrap, Journal of the Royal Statistical Society, Series B 56(1), 3-48. 
Paap, R. and Dijk, H. K. V.: 2003, Bayes estimates of Markov trends in possibly cointegrated series: An application to US consumption and income, Journal of Business and Economic Statistics 21(4), 547-563.

Phillips, K. L.: 1991, A two-country model of stochastic output with changes in regime, Journal of International Economics 31, 121-142.

Smith, P. and Summers, P.: 2002, Regime switches in GDP growth and volatility: Some international evidence and implications for modelling business cycles*, Melbourne Institute Working Paper.

Smith, P. and Summers, P.: 2003, Identification and normalization in markov switching models of "business cycles".

Stock, J. H. and Watson, M. W.: 2002, Has the business cycle changed and why?, in M. Gertler and K. Rogoff (eds), NBER Macroeconomics Annual, MIT Press. 


\section{Appendix A Parameter estimates}

\section{A.1 VAR}

$$
\begin{aligned}
& \widehat{\Pi}=\left(\begin{array}{rrrrrrr} 
& A U S & U S A & U K & G E R & C A N & J P N \\
A U S & -\mathbf{0 . 1 8} & \mathbf{0 . 1 6} & 0.04 & -0.05 & -0.01 & 0.03 \\
U S A & -0.02 & -0.07 & 0.06 & -0.04 & 0.00 & 0.03 \\
U K & 0.05 & 0.08 & -0.09 & -\mathbf{0 . 1 0} & -0.04 & 0.03 \\
G E R & 0.01 & 0.04 & 0.07 & -\mathbf{0 . 1 8} & -0.04 & 0.06 \\
C A N & 0.02 & -0.02 & 0.04 & -0.04 & -0.02 & 0.02 \\
J P N & -0.04 & \mathbf{0 . 1 6} & -0.02 & \mathbf{- 0 . 1 1} & -0.02 & 0.03
\end{array}\right) \\
& \widehat{\bar{\Phi}}=\left(\begin{array}{rrrrrrr} 
& A U S & U S A & U K & G E R & C A N & J P N \\
A U S & -0.04 & -0.17 & -0.08 & \mathbf{0 . 2 4} & 0.18 & 0.02 \\
U S A & 0.09 & \mathbf{0 . 2 6} & 0.09 & 0.02 & 0.05 & -0.01 \\
U K & -0.10 & 0.14 & -0.02 & -0.01 & 0.04 & -0.03 \\
G E R & -\mathbf{0 . 1 7} & \mathbf{0 . 2 8} & 0.11 & -\mathbf{0 . 1 8} & -0.03 & -0.06 \\
C A N & 0.08 & \mathbf{0 . 4 7} & 0.07 & \mathbf{0 . 1 1} & -0.02 & -0.11 \\
J P N & 0.04 & -0.03 & 0.11 & \mathbf{0 . 1 6} & -0.14 & -0.09
\end{array}\right) \\
& \widehat{N}_{1}=\left(\begin{array}{cc}
A U S & \mathbf{1 0 4 9} \\
U S A & \mathbf{7 7 8} \\
U K & \mathbf{1 1 2 7} \\
G E R & \mathbf{4 9 7} \\
C A N & \mathbf{3 2 9} \\
J P N & \mathbf{5 0 0}
\end{array}\right) \widehat{\Gamma}_{0}=\left(\begin{array}{cc}
A U S & \mathbf{1 . 2 7} \\
U S A & \mathbf{1 . 1 2} \\
U K & \mathbf{0 . 7 8} \\
G E R & \mathbf{1 . 1 0} \\
C A N & \mathbf{1 . 4 5} \\
J P N & \mathbf{2 . 5 1}
\end{array}\right) \\
& \widehat{\Sigma}=\left[\begin{array}{ccccccc} 
& A U S & U S A & U K & G E R & C A N & J P N \\
A U S & \mathbf{1 . 6 7} & 0.03 & 0.16 & 0.05 & \mathbf{0 . 2 4} & -0.11 \\
U S A & 0.03 & \mathbf{0 . 7 1} & \mathbf{0 . 1 8} & 0.01 & \mathbf{0 . 2 7} & 0.08 \\
U K & 0.16 & \mathbf{0 . 1 8} & \mathbf{0 . 9 6} & \mathbf{0 . 2 0} & 0.06 & \mathbf{0 . 2 0} \\
G E R & 0.05 & 0.01 & \mathbf{0 . 2 0} & \mathbf{1 . 4 2} & -0.02 & 0.19 \\
C A N & \mathbf{0 . 2 4} & \mathbf{0 . 2 7} & 0.06 & -0.02 & \mathbf{0 . 6 6} & 0.05 \\
J P N & -0.11 & 0.08 & \mathbf{0 . 2 0} & 0.19 & 0.05 & \mathbf{1 . 0 3}
\end{array}\right] .
\end{aligned}
$$




\section{A.2 MS-VAR}

$$
\begin{aligned}
& \widehat{\Pi}=\left(\begin{array}{ccccccc} 
& A U S & U S A & U K & G E R & C A N & J P N \\
A U S & -\mathbf{0 . 3 9} & -0.10 & 0.12 & -0.09 & -0.03 & 0.07 \\
U S A & 0.00 & -0.06 & 0.06 & -0.05 & -0.05 & 0.01 \\
U K & -0.03 & -\mathbf{0 . 2 8} & -0.03 & \mathbf{- 0 . 1 1} & 0.18 & 0.00 \\
G E R & 0.13 & 0.32 & 0.16 & \mathbf{- 0 . 1 8} & -0.25 & 0.06 \\
C A N & 0.10 & \mathbf{0 . 1 8} & 0.07 & -0.02 & -\mathbf{0 . 3 0} & 0.02 \\
J P N & 0.01 & 0.21 & 0.01 & \mathbf{- 0 . 1 1} & -0.02 & 0.04
\end{array}\right) \\
& \widehat{\bar{\Phi}}=\left(\begin{array}{ccccccc} 
& A U S & U S A & U K & G E R & C A N & J P N \\
A U S & 0.06 & -0.09 & -0.33 & \mathbf{0 . 3 4} & 0.12 & 0.06 \\
U S A & 0.06 & 0.02 & 0.00 & 0.04 & 0.04 & 0.02 \\
U K & -0.11 & 0.16 & -0.08 & 0.01 & -0.04 & 0.00 \\
G E R & -\mathbf{0 . 3 4} & -0.33 & 0.11 & \mathbf{- 0 . 2 2} & -0.04 & -0.12 \\
C A N & 0.03 & \mathbf{0 . 3 2} & 0.16 & \mathbf{0 . 1 5} & 0.04 & -0.01 \\
J P N & 0.01 & -0.21 & 0.14 & 0.13 & -0.02 & \mathbf{- 0 . 1 9}
\end{array}\right) \\
& \widehat{N}_{1}=\left(\begin{array}{cc}
A U S & \mathbf{1 0 4 8} \\
U S A & \mathbf{7 7 8} \\
U K & \mathbf{1 1 2 7} \\
G E R & \mathbf{4 9 6} \\
C A N & \mathbf{3 2 9} \\
J P N & \mathbf{5 0 0}
\end{array}\right) \widehat{\Gamma}_{0}=\left(\begin{array}{cc}
A U S & \mathbf{1 . 2 5} \\
U S A & \mathbf{0 . 8 1} \\
U K & \mathbf{0 . 5 4} \\
G E R & \mathbf{0 . 7 7} \\
C A N & \mathbf{0 . 9 4} \\
J P N & \mathbf{2 . 5 1}
\end{array}\right) \\
& \widehat{\Gamma}_{1}=\left(\begin{array}{ccccccc} 
& A U S & U S A & U K & G E R & C A N & J P N \\
A U S & \mathbf{- 1 . 5 8} & -0.45 & 0.37 & \mathbf{- 2 . 1 7} & -\mathbf{1 . 5 4} & -1.04 \\
U S A & \mathbf{1 . 2 2} & \mathbf{- 1 . 1 8} & \mathbf{1 . 1 5} & \mathbf{- 0 . 9 6} & \mathbf{- 1 . 1 0} & -1.07 \\
U K & \mathbf{3 . 5 4} & -0.75 & \mathbf{- 0 . 6 7} & \mathbf{3 . 6 1} & -0.34 & \mathbf{- 1 . 7 0} \\
G E R & \mathbf{2 . 2 6} & \mathbf{- 1 . 6 1} & \mathbf{0 . 6 5} & \mathbf{- 1 . 1 6} & 0.11 & \mathbf{2 . 2 4} \\
C A N & 0.55 & 0.02 & \mathbf{1 . 3 8} & 0.65 & \mathbf{- 1 . 5 7} & -1.35 \\
J P N & \mathbf{1 . 1 6} & \mathbf{- 0 . 6 9} & 0.31 & 0.33 & \mathbf{0 . 4 6} & \mathbf{- 4 . 2 5}
\end{array}\right) \\
& \widehat{\Sigma}=\left[\begin{array}{ccccccc} 
& A U S & U S A & U K & G E R & C A N & J P N \\
A U S & \mathbf{1 . 7 3} & -0.04 & 0.23 & 0.23 & 0.11 & -0.10 \\
U S A & -0.04 & \mathbf{0 . 4 4} & 0.09 & -0.03 & 0.04 & -0.04 \\
U K & 0.23 & 0.09 & \mathbf{0 . 6 4} & 0.01 & 0.07 & 0.04 \\
G E R & 0.23 & -0.03 & 0.01 & \mathbf{1 . 4 6} & 0.07 & 0.14 \\
C A N & 0.11 & 0.04 & 0.07 & 0.07 & \mathbf{0 . 3 4} & 0.06 \\
J P N & -0.10 & -0.04 & 0.04 & 0.14 & 0.06 & \mathbf{0 . 8 6}
\end{array}\right] \\
& \widehat{p}=\left(\begin{array}{cc}
A U S & \mathbf{0 . 9 8} \\
U S A & \mathbf{0 . 9 7} \\
U K & \mathbf{0 . 9 2} \\
G E R & \mathbf{0 . 9 9} \\
C A N & \mathbf{0 . 9 5} \\
J P N & \mathbf{0 . 9 9}
\end{array}\right) \widehat{q}=\left(\begin{array}{cc}
A U S & \mathbf{0 . 5 5} \\
U S A & \mathbf{0 . 7 6} \\
U K & \mathbf{0 . 6 0} \\
G E R & \mathbf{0 . 6 6} \\
C A N & \mathbf{0 . 6 8} \\
J P N & \mathbf{0 . 6 6}
\end{array}\right)
\end{aligned}
$$




\section{Appendix B Standard errors}

\section{B.1 VAR}

$$
\begin{aligned}
& \widehat{\Pi}=\left(\begin{array}{rrrrrrr} 
& A U S & U S A & U K & G E R & C A N & J P N \\
A U S & 0.05 & 0.06 & 0.06 & 0.06 & 0.03 & 0.02 \\
U S A & 0.03 & 0.04 & 0.04 & 0.04 & 0.02 & 0.02 \\
U K & 0.04 & 0.05 & 0.05 & 0.04 & 0.02 & 0.02 \\
G E R & 0.05 & 0.06 & 0.06 & 0.06 & 0.03 & 0.02 \\
C A N & 0.03 & 0.04 & 0.04 & 0.04 & 0.02 & 0.01 \\
J P N & 0.04 & 0.05 & 0.05 & 0.05 & 0.03 & 0.02
\end{array}\right) \\
& \widehat{\bar{\Phi}}=\left(\begin{array}{rrrrrrr} 
& A U S & U S A & U K & G E R & C A N & J P N \\
A U S & 0.08 & 0.14 & 0.11 & 0.09 & 0.13 & 0.11 \\
U S A & 0.05 & 0.09 & 0.08 & 0.06 & 0.08 & 0.07 \\
U K & 0.06 & 0.11 & 0.09 & 0.07 & 0.10 & 0.08 \\
G E R & 0.07 & 0.13 & 0.11 & 0.08 & 0.12 & 0.10 \\
C A N & 0.05 & 0.09 & 0.07 & 0.05 & 0.08 & 0.07 \\
J P N & 0.06 & 0.11 & 0.09 & 0.07 & 0.10 & 0.08
\end{array}\right) \\
& \widehat{N}_{1}=\left(\begin{array}{cc}
A U S & 1.11 \\
U S A & 0.79 \\
U K & 0.92 \\
G E R & 1.12 \\
C A N & 0.80 \\
J P N & 1.00
\end{array}\right) \widehat{\Gamma}_{0}=\left(\begin{array}{cc}
A U S & 0.17 \\
U S A & 0.16 \\
U K & 0.15 \\
G E R & 0.14 \\
C A N & 0.26 \\
J P N & 0.30
\end{array}\right) \\
& \widehat{\Sigma}=\left[\begin{array}{ccccccc} 
& A U S & U S A & U K & G E R & C A N & J P N \\
A U S & 0.20 & 0.09 & 0.11 & 0.13 & 0.09 & 0.11 \\
U S A & & 0.08 & 0.07 & 0.08 & 0.06 & 0.07 \\
U K & & & 0.11 & 0.10 & 0.07 & 0.09 \\
G E R & & & & 0.17 & 0.08 & 0.10 \\
C A N & & & & & 0.08 & 0.07 \\
J P N & & & & & & 0.12
\end{array}\right]
\end{aligned}
$$




\section{B.2 MS-VAR}

$$
\begin{aligned}
& \widehat{\Pi}=\left(\begin{array}{ccccccc} 
& A U S & U S A & U K & \text { GER } & C A N & J P N \\
A U S & 0.11 & 0.17 & 0.09 & 0.09 & 0.19 & 0.04 \\
U S A & 0.06 & 0.10 & 0.06 & 0.03 & 0.12 & 0.02 \\
U K & 0.07 & 0.09 & 0.07 & 0.05 & 0.12 & 0.02 \\
G E R & 0.11 & 0.18 & 0.09 & 0.06 & 0.20 & 0.03 \\
C A N & 0.06 & 0.08 & 0.06 & 0.05 & 0.10 & 0.01 \\
J P N & 0.07 & 0.12 & 0.05 & 0.05 & 0.12 & 0.02
\end{array}\right) \\
& \widehat{\bar{\Phi}}=\left(\begin{array}{ccccccc} 
& A U S & U S A & U K & G E R & C A N & J P N \\
A U S & 0.10 & 0.25 & 0.17 & 0.10 & 0.18 & 0.12 \\
U S A & 0.06 & 0.14 & 0.10 & 0.05 & 0.09 & 0.06 \\
U K & 0.07 & 0.16 & 0.11 & 0.06 & 0.10 & 0.07 \\
G E R & 0.10 & 0.25 & 0.15 & 0.09 & 0.17 & 0.10 \\
C A N & 0.05 & 0.13 & 0.09 & 0.05 & 0.08 & 0.05 \\
J P N & 0.08 & 0.18 & 0.12 & 0.07 & 0.12 & 0.08
\end{array}\right) \\
& \widehat{N}_{1}=\left(\begin{array}{cc}
A U S & 1.30 \\
U S A & 0.84 \\
U K & 1.07 \\
G E R & 1.31 \\
C A N & 0.86 \\
J P N & 1.07
\end{array}\right) \widehat{\Gamma}_{0}=\left(\begin{array}{cc}
A U S & 0.16 \\
U S A & 0.14 \\
U K & 0.26 \\
G E R & 0.21 \\
C A N & 0.18 \\
J P N & 0.30
\end{array}\right) \\
& \widehat{\Gamma}_{1}=\left(\begin{array}{ccccccc} 
& A U S & U S A & U K & \text { GER } & C A N & J P N \\
A U S & 0.23 & 0.40 & 0.38 & 0.89 & 0.24 & 1.27 \\
U S A & 0.36 & 0.18 & 0.18 & 0.39 & 0.17 & 0.78 \\
U K & 0.56 & 0.44 & 0.29 & 0.54 & 0.19 & 0.62 \\
G E R & 0.55 & 0.31 & 0.27 & 0.25 & 0.20 & 0.84 \\
C A N & 0.36 & 0.43 & 0.21 & 0.53 & 0.22 & 0.73 \\
J P N & 0.36 & 0.23 & 0.24 & 0.50 & 0.18 & 0.44
\end{array}\right) \\
& \widehat{\Sigma}=\left[\begin{array}{ccccccc} 
& A U S & U S A & U K & \text { GER } & C A N & J P N \\
A U S & 1.05 & 0.26 & 0.38 & 0.45 & 0.31 & 0.31 \\
U S A & & 0.14 & 0.13 & 0.18 & 0.12 & 0.11 \\
U K & & & 0.30 & 0.24 & 0.15 & 0.15 \\
G E R & & & & 0.55 & 0.24 & 0.28 \\
C A N & & & & & 0.23 & 0.14 \\
J P N & & & & & & 0.24
\end{array}\right] \\
& \widehat{p}=\left(\begin{array}{cc}
A U S & 0.01 \\
U S A & 0.01 \\
U K & 0.03 \\
G E R & 0.01 \\
C A N & 0.02 \\
J P N & 0.01
\end{array}\right) \widehat{q}=\left(\begin{array}{cc}
A U S & 0.18 \\
U S A & 0.11 \\
U K & 0.11 \\
G E R & 0.18 \\
C A N & 0.11 \\
J P N & 0.18
\end{array}\right)
\end{aligned}
$$

